Article

\title{
Is the Maker Movement Contributing to Sustainability?
}

\author{
Jeremy Millard ${ }^{1, *}$, Marie N. Sorivelle ${ }^{2}$, Sarah Deljanin ${ }^{1}$, Elisabeth Unterfrauner ${ }^{3}$ and \\ Christian Voigt ${ }^{3}$ \\ 1 International Center, Danish Technological Institute, DK-8000 Aarhus C, Denmark; \\ deljaninsarah@gmail.com \\ 2 Ideas and Innovation, Danish Technological Institute, DK-2630 Taastrup Copenhagen, Denmark; \\ mnse@teknologisk.dk \\ 3 Centre for Social Innovation, A-1150 Vienna, Austria; unterfrauner@zsi.at (E.U.); \\ voigt.cmc@gmail.com (C.V.) \\ * Correspondence: jeremy.millard@3mg.org; Tel.: +45-7220-1417
}

Received: 31 May 2018; Accepted: 26 June 2018; Published: 28 June 2018

\begin{abstract}
ICT has already revolutionized content creation and communications. In principle, today, everybody with Internet access, the right skills and equipment can produce digital content composed of virtual "bits" and make it instantly available across the globe. The same is now happening to manufacturing for everyone with access to tools like 3D printers. This inter-changeability of bits and atoms is being called the maker movement, which started as a community-based, socially-driven bottom-up movement but is today also impacting mainstream manufacturing through increased efficiencies, distributed local production and the circular economy. The maker movement thus has significant promise for increasing social, economic and environmental sustainability, but is it currently living up to this potential? This paper reports on work undertaken by the European-funded MAKE-IT project has examined this question through detailed qualitative and quantitative empirical research, including ten in-depth case studies across Europe and a detailed examination of 42 maker initiatives at Europe's foremost city-based maker faire, supplemented by extensive secondary research. Despite the maker movement's short history, the overall results provide sound evidence of its important though variable contribution to sustainability thus far. In addition, there is a strong gender dimension showing that females are underrepresented both as users and leaders of maker initiatives, whilst female leaders tend to achieve much higher sustainability impacts than their male counterparts. There is also clear evidence that maker initiatives in close collaboration with each other and other actors in city- and region-wide ecosystems are much more successful in achieving sustainability impacts than others.
\end{abstract}

Keywords: maker movement; digital fabrication; social sustainability; environmental sustainability; economic sustainability; gender; scale; quantitative analysis; qualitative analysis; case studies

\section{Introduction}

This objective of this paper is to examine the question of whether the maker movement is living up to its purported potential to contribute to increasing social, economic and environmental sustainability.

\subsection{The Context}

The maker movement is a rapidly expanding field with innumerable perspectives, interpretations and definitions. It is part of a major transformation in manufacturing towards distributed digital fabrication that potentially marks a decisive shift away from a society reliant on by mass production 
and consumption towards a society also characterized by "mass customization". Information and Communication Technology (ICT) has already revolutionized content creation and communications and has now become the general purpose technology underpinning all other technologies. In principle, today, everybody with Internet access, the right skills and equipment can produce digital content composed of virtual "bits" and make it instantly available across the globe. The same is now happening to the fabrication of physical objects for everyone with access to tools like 3D printers. This inter-changeability of bits and atoms is being called the maker movement, which started as a community-based, socially-driven bottom-up movement but is today also impacting mainstream manufacturing through increased efficiencies, distributed local production and the circular economy.

In principle, digital fabrication allows every organization and every individual to customize the products and services they consume. It is claimed that this new business model cuts waste and enables manufacturers to restructure supply chains and completely reorganize their businesses and operations [1-4]. Localised bespoke production becomes possible attuned to specific user requirements, in a step away from consumerism and towards "pro-sumerism", thereby also reducing environmental impacts because products can be produced close to where they are needed using largely re-cycled local waste materials as feedstock. Unusually for a manufacturing innovation, momentum started at the bottom, pioneered by non-profit makers who hack products and designs in addition to software. This also has significant social impacts such as spawning new skills and jobs and supporting community cohesion and promoting greater personal satisfaction, for example through the Fab Lab network (https://www.fablabs.io/labs/map). This blurs the relationships between producer, supplier and consumer, and also has profound implications for supply chains as well as for the organization and content of work, which will in turn have impacts on governance, regulation, education and social security. It is also claimed that the movement reflects cultural shifts enabling people to "reconnect hand with brain" and to "get their hands dirty again" [5], thereby providing sustainability benefits across the social, environmental and economic spectrums.

\subsection{Defining the Maker Movement}

The maker movement is a rapidly expanding field with innumerable perspectives, interpretations and definitions. In the specific context of the MAKE-IT project (2016-2017), the definition of the maker movement focuses on the overlap between four main fields of activity (Figure 1).

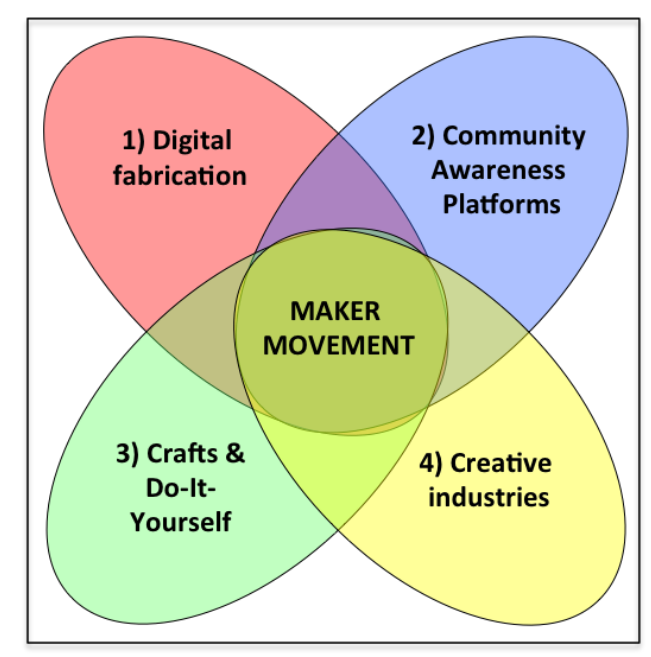

Figure 1. The four components of the maker movement.

\subsubsection{Digital Fabrication}

Digital fabrication provides the technological underpinning of the Maker movement. Digital modeling and fabrication is a process that unifies design with production through the use of $3 \mathrm{D}$ 
modeling software or computer-aided design (CAD) and additive and subtractive manufacturing processes. $3 \mathrm{D}$ printing is a type of additive fabrication, whilst machining is a type of subtractive fabrication. The initial focus on simple 3D printers has now progressed to an awareness that the real maker revolution comes when these tools are combined with laser cutters, precision mills, large and small format mills, actuators, sensors, as well as digital assemblers and re-assemblers. These use various combinations of feed-stocks, including biological materials as well as pulverized, sintered or melted plastic, rubber, metal, glass, wood, ceramics, paper, etc., much of which is very inexpensive and sourced locally $[1,3]$.

Tools and materials like these used together give makers at any scale and size a whole suite of capabilities for additive and subtractive manufacturing. They are being installed and used, not just in industry and research labs, but in many communities by Small and Medium Sized-Enterprises (SMEs), start-ups, civil organizations and even individuals. As in the ICT revolution over the last twenty years, their costs are tumbling fast and their quality and ease of use are dramatically improving [2], and just like its digital predecessor that saw disruption driven by personal computers and co-creation online, much of the energy and innovation of the digital fabrication revolution is being driven from the bottom. Thus with suitable feed-stocks, digital fabrication tools allow designers to produce both inert and bio-material objects from a digital source, as well as convert a material object back to digital format in a two-way process which can be shared both online and in situ. In this light, the new world of digital fabrication is indeed a cornerstone of the emerging so-called fourth industrial revolution [6,7].

\subsubsection{Community Awareness Platforms (CAPs)}

Communities such as the makers are building and exploiting communication infrastructures for collaboration, sharing and learning purposes. CAPs is the European Commission's initiative for designing and piloting online platforms that create awareness of sustainability problems and offer collaborative solutions (https://capssi.eu). These are based on networks (for example of people, ideas and sensors), thereby enabling new forms of social innovation that aim to support behavioral change, reputational processes and self-regulation to the maximum degree so that these are trustable and effective. These can express themselves, for example, in new lifestyles and in consumption and production patterns, and give power not only to for-profit platforms but also to those which have, at least in part, non-commercial objectives. CAPs use ICT tools and networks for supporting and propelling new forms of sustainability and social innovation. This is done through a people-centric approach that aims to actively involve citizens in creating multi-dimensional communities at the grassroots, whilst at the same time linking into wider social, economic, environmental and democratic systems [8].

CAPs are described as mobilizing the network effect of collective intelligence for the public good, where this is seen as expressing itself through such phenomena as crowdsourcing and connective communities, and is often the result of forms of bottom-up and self-organization [9]. In this context, the concept of CAPs has arisen from three trends, each of which can harness and deploy different forms of collective intelligence, and lead to better policies and actions in tackling the many societal challenges we are facing. All three can lead to what is termed "hyper-connected humanity", the ultimate goal being to foster a more sustainable future based on a low-carbon, beyond-GDP economy, and a resilient, cooperative democratic community: first, social networking; second, direct contact with the environment through the Internet of Things; and third, the collaborative production of knowledge, like Wikipedia [9]. A fourth major trend can now be added to this repertoire, i.e., not only the collaborative production of knowledge and other forms of intangible content, but also the collaborative production and consumption of tangible forms of physical objects, as currently being realized by the burgeoning maker movement. 


\subsubsection{Crafts, Do-It-Yourself, Creative and Learning Activities}

The tradition of craft production is the process of manufacturing by hand with or without the aid of advanced or power tools. In parallel with this, do-it-yourself presents gateway opportunities for the un-skilled or novice to build, modify or repair something without the direct aid of experts or professionals. Both can also express themselves by developing an ethos of self-help, learning and competence building, often in shared and collaborative spaces like libraries, repair cafés, schools, universities and other common, shared or public spaces. Such spaces are often aimed at specific target groups, such as those who are marginalized or vulnerable in some way, and support hands-on experiences and development and production of creative ideas. Many become "constructionist learning environments" for building "social-emotional competences" with strong social-political potential, social capital and social inclusion [10].

According to Charney [5], the idea of "making" is more important than seeing people primarily as "makers", given that making is taking place as just one activity intimately mixed/bundled with other activities. This emphasizes the notion that the maker movement is not a discrete, separate phenomenon but instead points to a broader "making culture" reflecting the burgeoning desire amongst many people to move on from a purely consumerist society to start again "getting their hands dirty". This means there is a need to think less about promoting and supporting a discrete separate "maker culture", and more about a broad and integrative "making culture". Thus, it is important to move away from only seeing making as an instrumental activity for people to make their own products with the focus largely on the technology, towards also supporting a more widespread change of people wishing to "reconnect hand and brain". In practice this also means, for example, not establishing making only as a separate activity in separate maker spaces, but ensuring making is an integral part of the wider range of activities in many institutions and premises, such as libraries, educational institutes, shopping centers, repair shops, community facilities of all kinds, etc. Thus, maker spaces in this context focus on encouraging "trying", "doing", "creating spontaneously", "enjoying" and "having fun" [5]. The very essence of maker spaces lies in creativity, informality, doing things without pressure and a "try-and-fail-and-try-again" approach. In this way there is often an ethical and inclusive imperative in making things, nurtured through capacity building and empowerment, exploration, experimentation, fun and the absence of pressure to excel, coupled with sharing and gaining new knowledge [10,11].

\subsubsection{The Creative Industries}

Arising from the craft and do-it-yourself cultures but distinct from these, many makers are today overlapping and working with the so-called creative industries as creative, arts and culture-based economic activities. These constitute a range of commercial activities which are concerned with the generation and exploitation of knowledge derived from, for example, architecture, art, cultural heritage, crafts, design, fashion, film, music, the performing arts, publishing, research and development (R \& D), software, toys and games, TV and radio and video games. ICT tools are playing a significant role in boosting these industries as they enable, often for the first time, any actor to collect, preserve, organize and distribute creative and cultural content, ranging from languages to historical artifacts. Makers are providing much of the new physical and digital inputs to these industries, many of which are still small scale, often recently established as start-ups, social entrepreneurs or SMEs, but also increasingly working with and becoming larger enterprises, both internationally as well as locally.

The creative industries employed 11.4 million people in the European Union (EU) in 2015, accounting for $5 \%$ of the workforce, and this number is rising fast. They are characterized by higher than average levels of life satisfaction and wellbeing, with the largest number of jobs being ICT-related: "computer consultancy and programming activities" [12]. Creativity is also inversely related to computerizability: $87 \%$ of highly creative workers are at low or no risk of full automation, compared to $40 \%$ of jobs in the UK workforce as a whole. Such findings reflect the fact that machines can most successfully emulate humans when a problem is well specified in advance-that is, when performance can be straightforwardly quantified and evaluated-and when the work task 
environment is sufficiently simple to enable autonomous control. Machines struggle when tasks are highly interpretive, geared at products whose final form is not fully specified in advance and when work task environments are complex-a good description of most creative as well as maker occupations [13].

\section{Materials and Methods}

An overview of the methods and materials deployed for mapping and analyzing the maker movement in Europe is provided in Figure 2. This sketches how the overall analytical framework interacts with two main research instruments. The former consists of three analytical pillars: (1) organization and governance; (2) collaborative behaviors and (3) sustainability impact examining social, economic and environmental sustainability. The framework provides a common template for guiding the two research instruments: qualitative in-depth case studies, and a quali-quantitative survey. More details are given below.

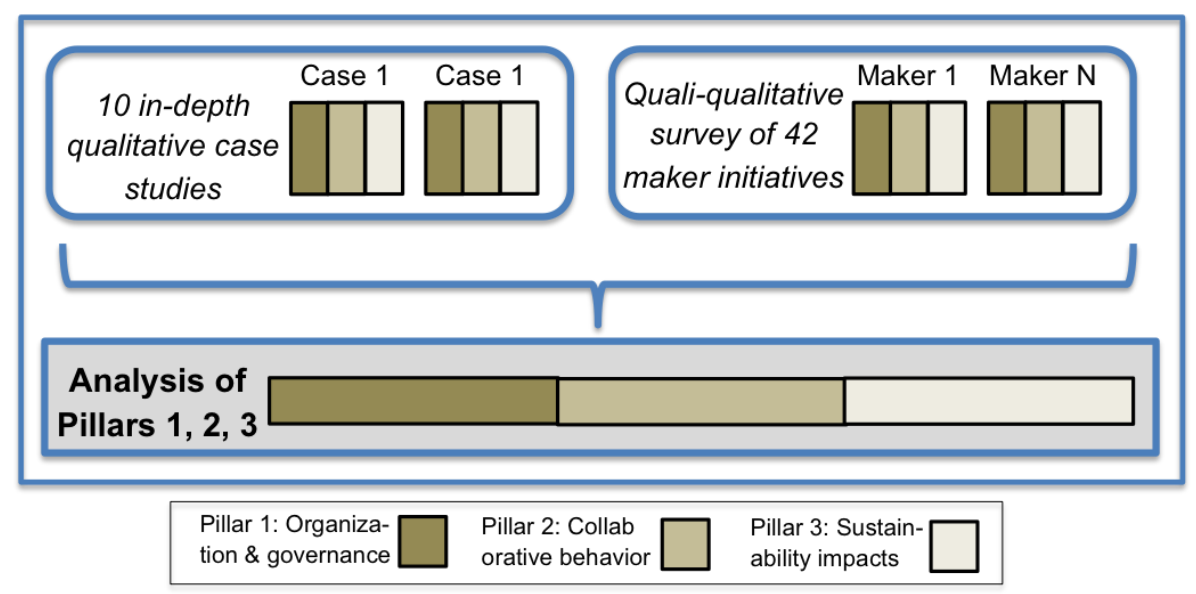

Figure 2. Overview of methodology and tools used for analysis.

\subsection{Three Analytical Pillars}

The analytical framework consists of three analytical pillars for examining individual maker initiatives and making comparisons between them: (i) how maker communities are organised and governed; (ii) what maker participants do and how they behave through peer and collaborative activities; and (iii) the various ways maker initiatives impact on, and add value to, society with particular reference to their sustainability impacts. (See Figure 3). The three pillars are ambitious in scope, and have also been shown in the project to be theoretically sound as well as highly robust empirically when examining both the internal features and the external relations and interactions of maker initiatives. Additionally, they are able to guide the examination of secondary evidence from the academic and grey literature in support of empirical analysis. As illustrated in Figure 3, the three pillars are designed to provide a comprehensive but also simple set of insights which show both the "means" by which maker initiatives operate and the "ends" such "means" produce.

\subsubsection{Organization and Governance}

Pillar 1 examines how maker communities are organised and governed, including strategies and financing, supply chains, management of knowledge and innovation, the institutional environment as well as gender issues and social responsibility. Pillar 1 looks at the ways that maker communities using CAPs are organised both internally and externally, the legal and regulatory frameworks that promote or retard them, their IPR (Intellectual Property Right) implications, security, safety and privacy issues, and the interfaces they have with their institutional and policy environments which include social, economic, environmental and technological systems. 


\subsubsection{Peer and Collaborative Behaviors}

Pillar 2 examines what maker participants do and how they behave, including the processes of social engagement and social influence, ethical issues, participation, collaboration, learning, sharing, community forming and inclusive approaches. Pillar 2 looks at the mechanisms and activities, including generating awareness and leveraging peer pressure. It examines what drives people's behaviors to take-up maker activity and/or establish or join a maker community, and how this supports better lifestyles through behavioral and system change. These activities and behaviors include learning, sharing, collaborating and realizing new forms of production, including social, economic, environmental and technological issues.

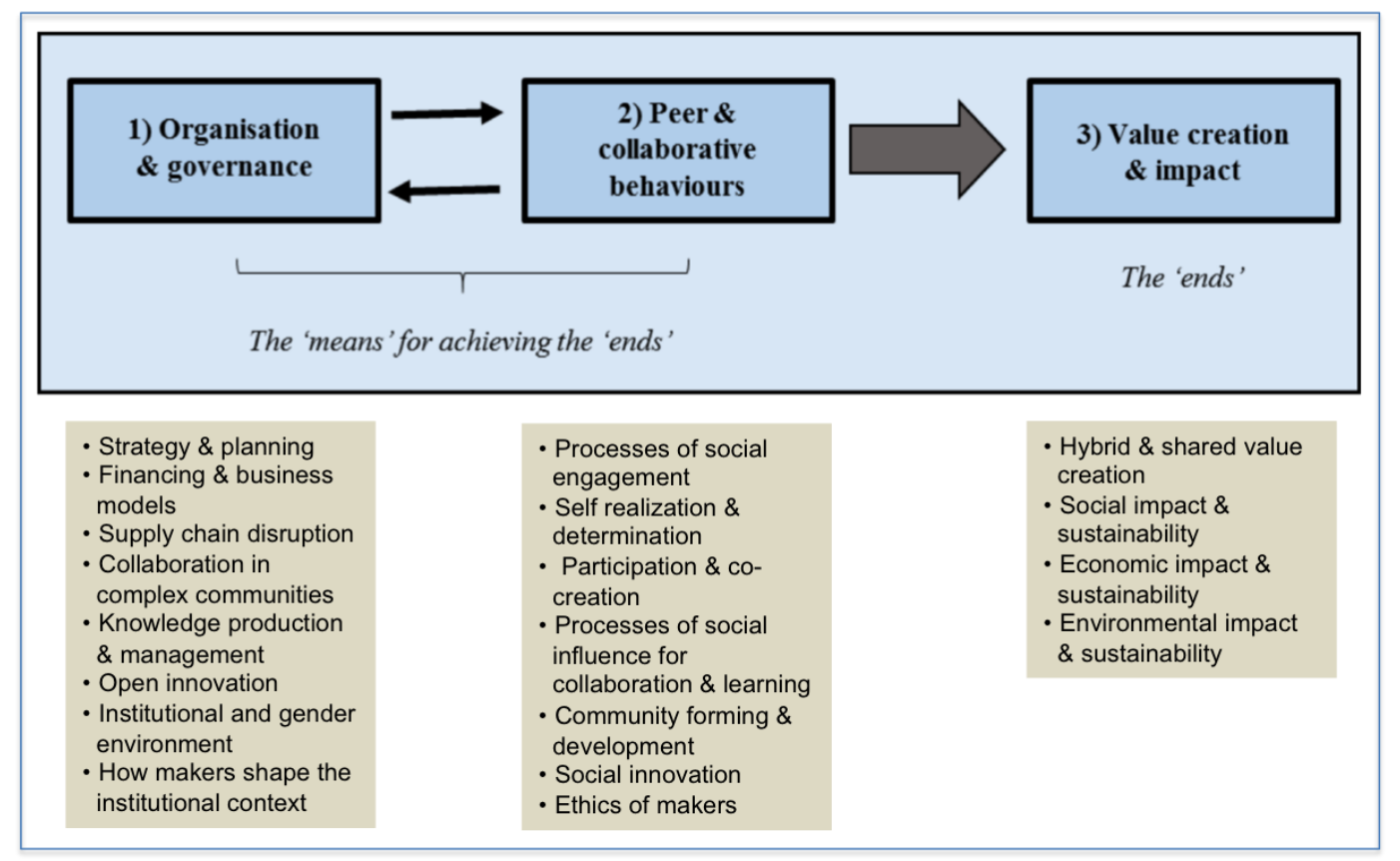

Figure 3. The three analytical pillars of MAKE-IT.

\subsubsection{Value Creation and Impact}

Pillar 3 examines the various ways interactions between pillars 1 and 2 impact on and add value to society: social, economic, environmental and hybrid value and impacts, with a strong focus on sustainability issues. Pillar 3 looks at the ways and extent to which maker communities create and capture such value and sustainability impacts, including through new forms of local, bottom-up business-, social- and sustainable-models, transversing between non-monetized and monetized accounting frameworks as well as different impact assessment methods. Local, national and global development and sustainability issues are examined. An historical perspective is also adopted, for example in analyzing the maker movement's trajectory in the 21st century as compared to the late 20th century development of personal computing and other technology innovations. This should also be seen in the context of today's industry increasingly moving away from centralized mass production and consumption towards decentralization and the mass customization of production and consumption.

\subsection{Two Research Instruments}

Using the analytical framework, two main research instruments were deployed, partially derived from the literature cited above plus widespread consultation. 


\subsubsection{Ten In-Depth Qualitative Case Studies}

Ten in-depth qualitative case studies from across Europe were undertaken, based both on background desk research on each case plus a set of structured open questions for interviewing and analysis. A minimum of four interviews per case was undertaken, i.e., the leader and three users. The cases were selected to reflect the range of types and configurations of maker activities in Europe along two dimensions as shown in Figure 4:

1. The vertical $y$ axis (marked in yellow) shows the scale and interaction dimension of maker initiatives. This ranges from "situational awareness" where maker initiatives tend to be relatively isolated, unconnected and focused mainly on specific or local areas, to "distributed awareness" where maker initiatives tend to be strongly networked, interconnected and collaborative over typically large and extensive areas. A number of positions were hypothesized along this dimension each of which was substantiated by the evidence subsequently collected. These were: (a) single/individual makers; (b) makers with a limited set of regular partners; (c) medium-scale communities of makers and their users; (d) maker ecosystems of interacting, diverse but complementary makers including other relevant actors; and (e) maker networks which tend to be very large scale, often national or international in extent, consisting of maker relationships built on common needs and interests, for example for mutual learning.

2. The horizontal of $x$ axis (marked in blue) shows the social innovation dimension derived from BEPA [14] and as described by Hochgerner [15] and Millard et al. [16]. This shows three types of social innovation.: (a) social demand as the narrowest view of social innovation outcomes focused on tackling problems in specific, possibly disparate, parts of society on a one-by-one, perhaps even piecemeal, basis; (b) societal challenge innovation at the boundary where the social, the economic and the environmental become blurred, as all actors can be involved, that are directed towards outcomes across society as a whole, and where an important focus is on the relationships between actors and outcomes; and (c) systemic change innovations that contribute, often together and cumulatively, to the reform of underlying structures, relationships and governance in society

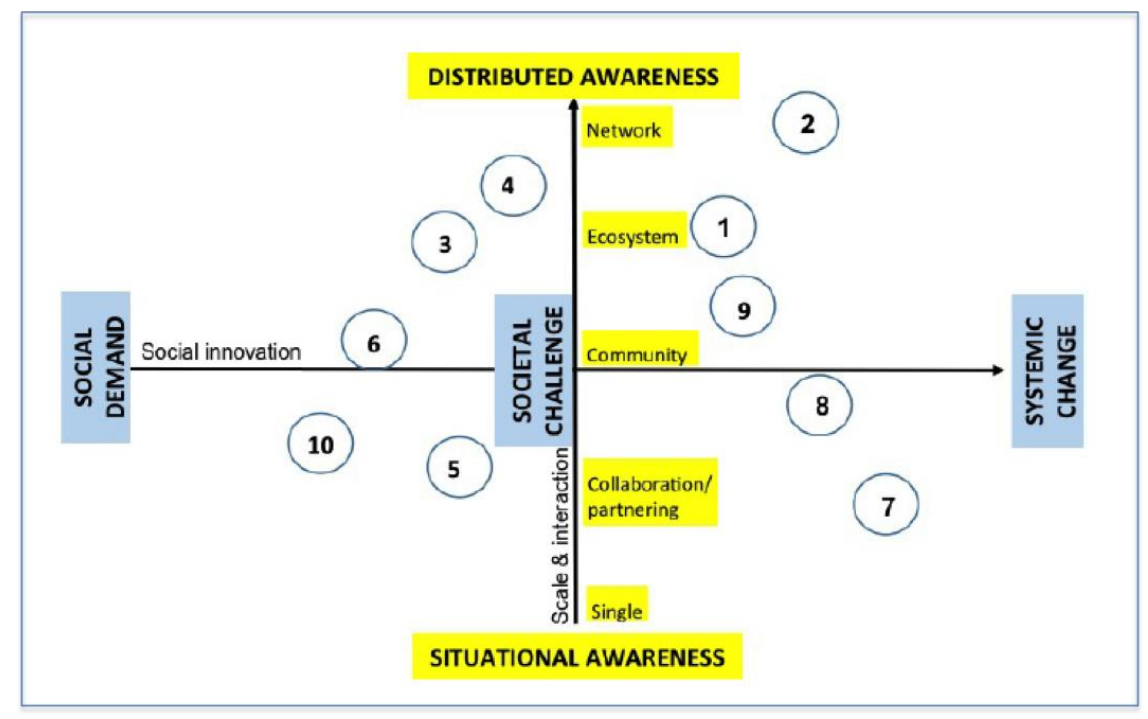

Figure 4. Selection of the ten MAKE-IT case studies.

Figure 4 sketches the placements of the ten maker case studies across these two dimensions: (1) Danish Technological Institute Lab, Denmark-a metropolitan FabLab; (2) Fablab Barcelona, Spain - a metropolitan FabLab and the central node of the Global FabLab Network; (3) Arduino, Italy—open source micro-computing hardware; (4) Smart Bending Factory, the Netherlands—open 
factory and regional metalworking network; (5) Mini Maker Faire Tartu, Estonia-a local maker faire; (6) Happylab Vienna, Austria-focused on low-threshold access, empowerment and inclusion; (7) Dezentrale, Germany—urban neighborhood FabLab; (8) Hochschule Ruhr-West Lab, Germany-manufacturer of maker technology; (9) Create It Real, Denmark; and (10) Fablab Zagreb, Croatia-NGO FabLab.

\subsubsection{Survey Questionnaire with Quali-Quantitative and Open Questions for Large Scale Survey}

This second research instrument supplements the first by using a common set of open qualitative questions, plus 7-point Likert scale propositions for completion during conversations between a maker initiative and an independent expert. This scale ranges from completely disagree with a given statement (for example "has the initiative improved human capital, such as skills and competences"), through mainly and then partially disagree, neither disagree nor agree, then to partially, mainly and completely agree. A "not applicable" option was also available. The Likert questions rely on self-evaluation, but the results were validated during the conversation between the respondent and the independent expert so that the latter could request justification and evidence for the score proposed to ensure as much objective accuracy as possible. The Likert scores, being both negative as well as positive in relation to the proposition, were summed and standardized across all answers based on these negative and positive weights, to provide generalized findings across the sample population or selected segments of it for comparison purposes. A "relevance" score was also derived for each proposition, calculated by deducting the percentage of interviewees stating that a particular question was "not applicable" from 100\%.

\subsection{Applying the Research Instruments to the Analytical Framework}

The second instrument provides data on the types and leadership of maker initiatives, the technology used, their ambitions and their actual achievements across the three analytical pillars. The first instrument complements these largely "what" data with more detailed information on "how" and "why" the reported results might have been achieved. For example, the second instrument indicates gender gaps and differences, which is confirmed by the first instrument adding some qualitative understanding, e.g., the "genderization" of tasks and objects in the maker space, differences in the use of technology, etc. [17].

The survey questionnaire was used to undertake structured interviews with a sample of 42 maker initiatives present at the Barcelona Maker Faire, 16-18 June 2017, out of 82 registered as present. Given that the initiatives were organized according to their main purpose and focus across the floor of the exhibition space, every second initiative was interviewed, thereby ensuring a representative sample. The Faire consisted of a highly diverse set of exhibitors drawn from across Barcelona and Catalonia in north-east Spain. European experts on the MAKE-IT team conducted the interviews and validated that the Barcelona maker scene is quite typical of other large cities across Europe.

When using the survey instrument in Barcelona, only one person was interviewed in most cases, although they were requested to speak on behalf of the initiative as a whole and not their own personal position or interests. Gender and age were, as far as possible, recorded referring to the main leader and/or practitioner rather than the manager/administrator or interviewee, if different. In most cases, the actual leader was interviewed as the faire was considered important enough that they should be present in person. The dataset has the potential for many more analyses, for example looking for comparisons and correlations between different variables. There are, as always, issues about the representativeness and validity of these data. However, the results from the two different sources do tend to corroborate each other in areas where there is overlap, and much care has been taken in data collection and preparation. All data and instruments are available on the MAKE-IT website in open format to support ongoing research (http://make-it.io/open-data-api/).

Both instruments went through various iterations of development, piloting and testing, and are best deployed together and alongside desk research as well as by making comparisons with other 
studies. The result provides a "quali-quantitative" analysis of the maker movement in Europe, anno 2017, the first of its kind to the authors' knowledge that includes a quantitative analysis of maker initiatives based on a reasonably sized sample. Given lack of space, only some headline results are presented in Section 3 below, plus cross-comparisons with gender and scale variables, particularly drawing out some sustainability issues.

\section{Results}

This section presents and interprets some of the findings of MAKE-IT's research using the analytical framework and research instruments described above.

\subsection{Gender and Age of Maker Leaders}

It is clear from Figure 5 that most maker initiatives are led by young males and that this is quite similar to the situation seen in the majority of technology-based start-ups and new enterprises. Female leaders constitute only $26 \%$ of all maker leaders in the Barcelona sample. These gender and age distributions are also in line with the results of the ten MAKE-IT case studies from across Europe, i.e., the majority of maker managers are male ( 9 out of10), while the gender ratio among users varies from 80:20 to 60:40, and most are relatively young, between 20 and 40 years of age. Again, this gives some confidence that the samples reflects maker initiatives more widely.
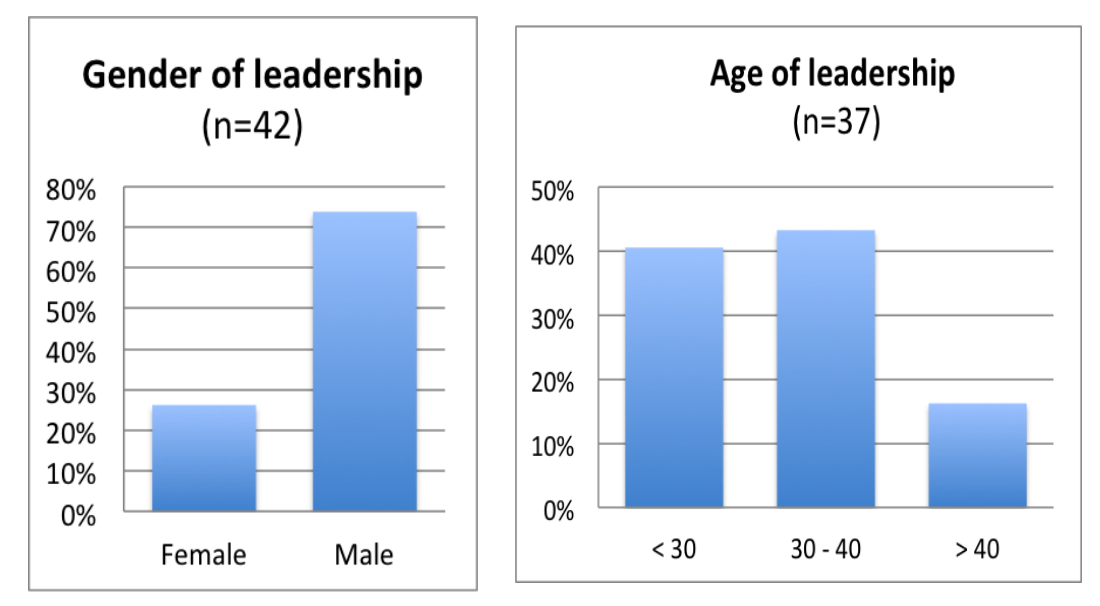

Figure 5. Gender and age of maker initiative leaders.

\subsection{Scale and Interaction of Maker Initiatives}

Based on the five scales that individual maker initiatives operate at, as described above, Figure 6 indicates that there is a good spread of maker scales, ranging from single initiatives that are more or less operating without significant interaction with other actors (which is the least common scale), up to makers operating in networks (the second least common). The overall distribution of makers in the sample starts to resemble a normal curve indicating that, at least on this characteristic, the sample seems to be relatively representative of makers generally. This is useful corroboration that this deductively derived scale range seems also to have relevance in practice. It is also clear from the second graph in Figure 6 that males and females are operating at quite different scales. Females are much more likely to work at the two extremes of the scale range, i.e., at the larger scales of ecosystems and networks and the smaller scale of singles, although they are also quite well represented in communities.

The case studies also demonstrate gender differences, including that female makers tend to work on different tasks "Woman make bio stuff; men make 3D prints" (maker manager, Germany) and on different projects and machines (while 3D printers are mostly used by men, plotters are more likely to be used by females). There is no equal participation of male and female makers in the cases and many 
interviewees lack female role models: "I do think that by having the Fab Lab attended by a girl, it makes other women interested in coming to the Fab Lab too" (maker manager, Denmark). This aligns with Kanter's theory of homo-social reproduction according to which people are more likely to find their ways into social environments if people with similar interests are already participating [18]. Role models are needed not only on a management level but also among facilitators in the maker space: "The problem is that if it's a man who is teaching, he would say it in one way, and a girl would say it in another way. They have role models, so if it is a woman saying it, it is great to look up to her and who she is. It really depends on who is standing there" (maker, Denmark). Interviewing maker managers, however, also had an impact on their reflection of male dominated cultures: "The visibility of women we never thought of, now it's on our minds. For some time now we have started thinking in these terms-how many girls we have, etc." (maker leader, Spain).
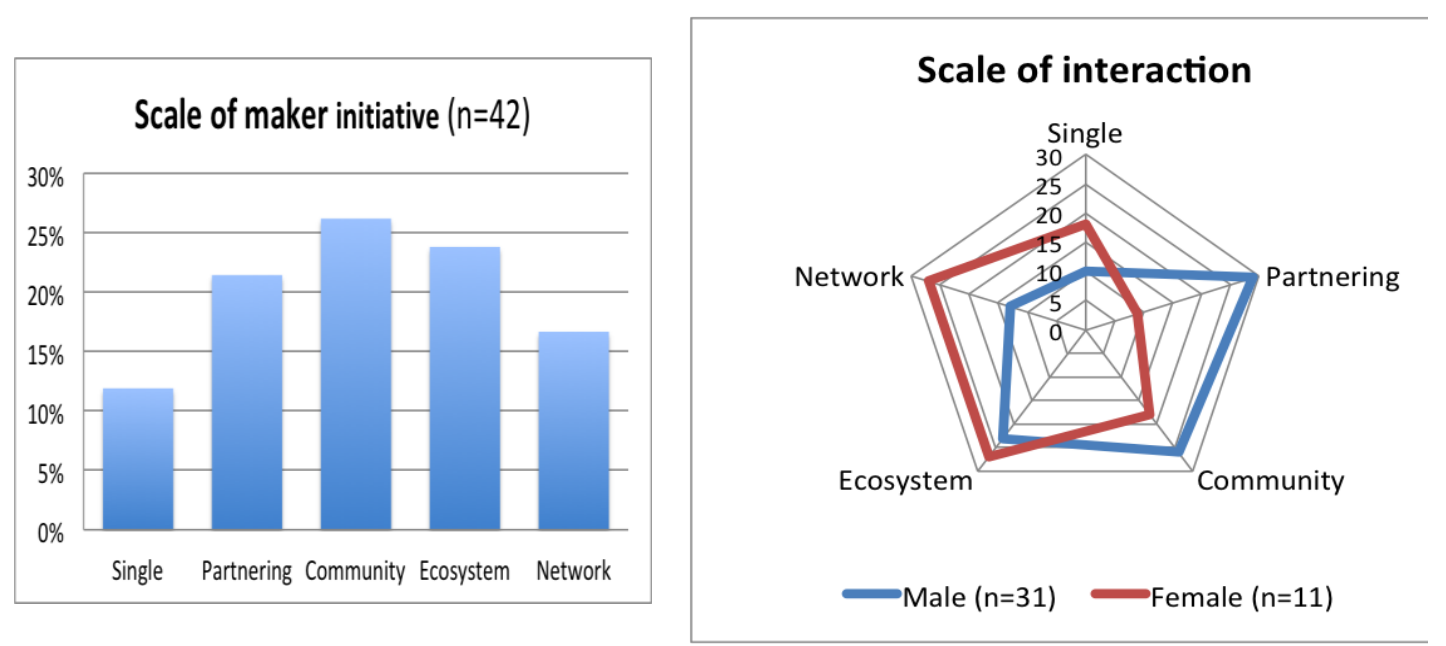

Figure 6. Scale and gender of maker initiatives.

\subsection{Technology Types and Technology Use}

The two diagrams in Figure 7 provide an overview of which types of technologies are used by both male- and female-led makers in the sample, as well as the purposes for which they are used. In the lefthand diagram of Figure 7, it is unsurprising that 3D printing technologies are the most common, given that these are, by and large, what defines who makers are. However, a large number of other technologies are also used, by far the majority of which are not specifically offered to or designed for makers. However, they are clearly maker-relevant and are being used quite intensely by makers, even though they are generally available technologies used across many sectors, by many different actors and for a multitude of purposes.

This conclusion has also been reached through a separate analysis of the technologies selected and described for makers in MAKE-IT'S TechRadar (http:/ / make-it.io:8080). In terms of the uses to which these technologies are put, again there is a wide spread, based on the categories proposed in the TechRadar. The least used categories are organization and communication which are more indicative of pillar 1 (organization and governance) activities. Indeed, other evidence presented below shows that pillar 1 has less focus in the sample and is less developed than pillar 2 (peer and collaborative behavior), perhaps because makers are generally still at quite an informal stage. This is also indicated by the fact that commercialization uses are only at rank 5 out of 8 in Figure 7, given that becoming commercial tends to take a long-term effort. These differences are not stark, however, and may also arise because many of the individuals interviewed in the Barcelona sample were lead practitioners rather than management or administrative staff. All the other technology uses are well represented and appear to be highly relevant for making activity. 

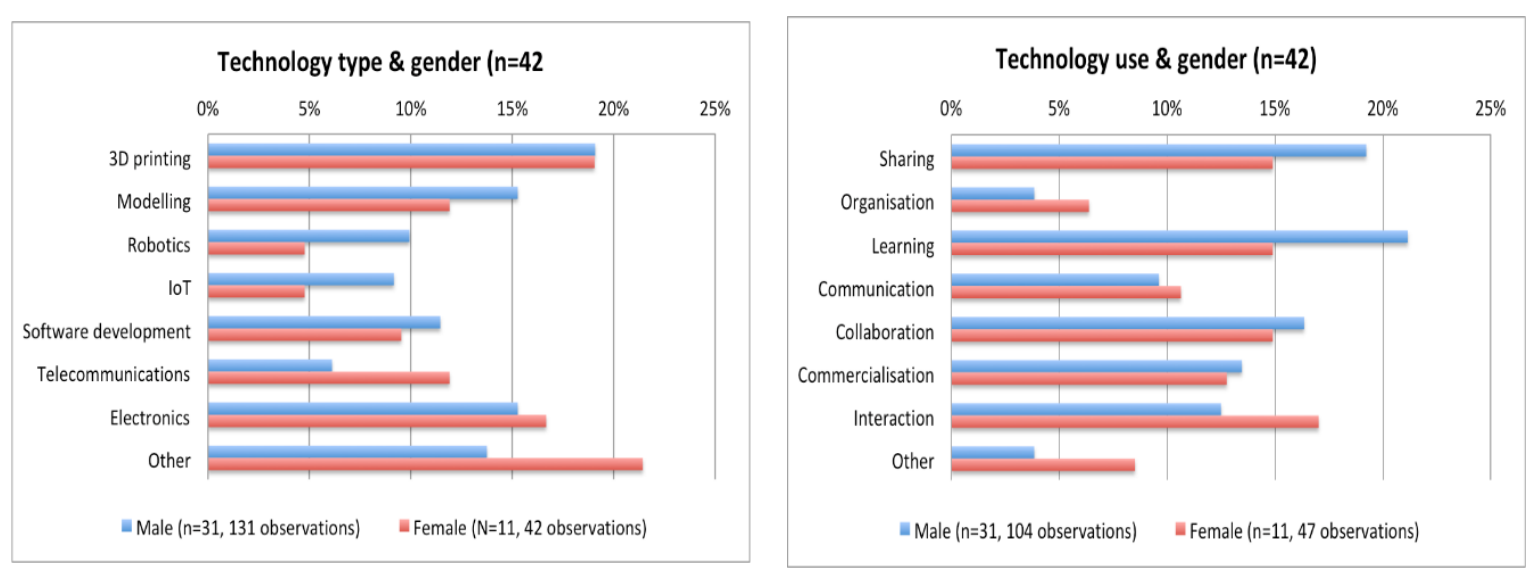

Figure 7. Technology type and use by gender.

It is also relevant to examine technology types and uses by gender, as also shown in Figure 7. The overwhelming conclusion is, in fact that both males and females generally use the same technologies to a similar extent. However, Figure 7 does indicate that males are a little more likely to be involved in modeling, software development, robotics and the Internet of Things (IoT), each of which is perhaps a more specialist technology in the maker context. Generally, females tend to use a wider range of technologies and to be less specialized in their technology use, as also indicated by the higher incidence of the "other" category.

In terms of technology use by gender, Figure 7 again indicates quite similar usage patterns between males and females, with a strong emphasis on sharing, collaboration, learning and interaction which are also more typical of pillar 2 as opposed to pillar 1 activities. However, there is one important exception, i.e., learning is much more important for males, whilst interaction is much more important for females. This observation seems to be in line with the fact that females are more likely than men to be leading maker initiatives at the larger scales, i.e., networking and ecosystems, as noted in the second graph in Figure 6. Men are also very marginally more likely to be using the technology for commercial purposes than females, and again females tend to be more generalist in their technology use as also indicated by the higher incidence of the "other" category.

Looking at the technology types at different scales, Figure 8 (on left) shows only a few distinctions. Most are used more or less equally at all scales, although there is a clear tendency for partnering to use 3D printing, modeling and other types more, and electronics, robotics and IoT less, when compared to many of the other scales. Examining how the technologies are used (Figure 8 on the right), however, shows some stronger distinctions. It seems clear that singles are quite random in their technology use given they can be highly diverse but perhaps also because their sample size is the smallest at 5 initiatives. The other noteworthy observation is that it is mostly in relatively small-scale partnerships that commercialization is taking place, whilst commercial uses of the technology are much less common at the larger scales of community, ecosystems and networks. Partnering is perhaps better able to provide the close bonding and trusting relationships necessary for makers to set up good commercial relationships in the maker context than are the other scales.

Also, as noted above, such commercial relations seem to focus more on 3D and modeling technologies, which is maybe unsurprising given that these are the original core technologies of the maker movement, so have had much more time to support commercial activities. It is also relevant to note that the largest scales to be more "all-round" in their use of technology, which again is perhaps not surprising as they involve many more actors and are generally more complex and comprehensive in the range of their activities. 

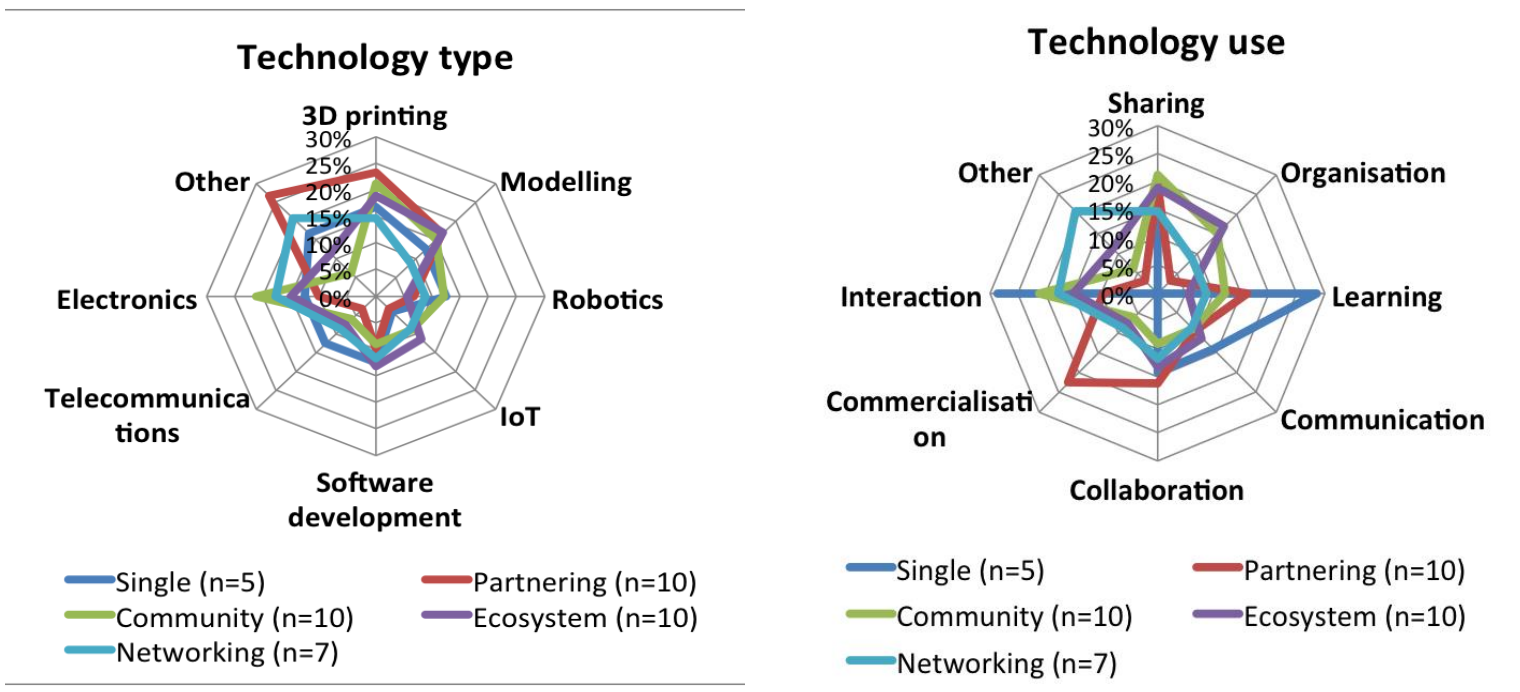

Figure 8. Technology type and use by scale.

\subsection{Long Term Ambition}

In Figure 9, the long-term ambitions of maker initiatives, as opposed to the actual achievements (which are discussed below), is charted. The two graphs show a distinction between an ambition being actively pursued (on the left) and how the interviewee sees the relevance of the ambition (on the right). The relevance scores are the percentages of initiatives indicating a given ambition is potentially relevant to them, whilst the ambition scores are those they are actually pursuing. As would be expected, in all cases the relevance scores are higher than the scores for ambitions actually being pursued, whilst the gap between the two indicates the size differentials of what is happening in practice and aspirations still to be tackled. So, for example, the gap between technical and educational science and research ambitions and their perceived relevance is much smaller than between commercial, environmental and social ambitions and their relevance. Clearly, there is more progress in terms of the former ambitions than the latter, and that the former are more indicative of the early stage of maker development than later stages.
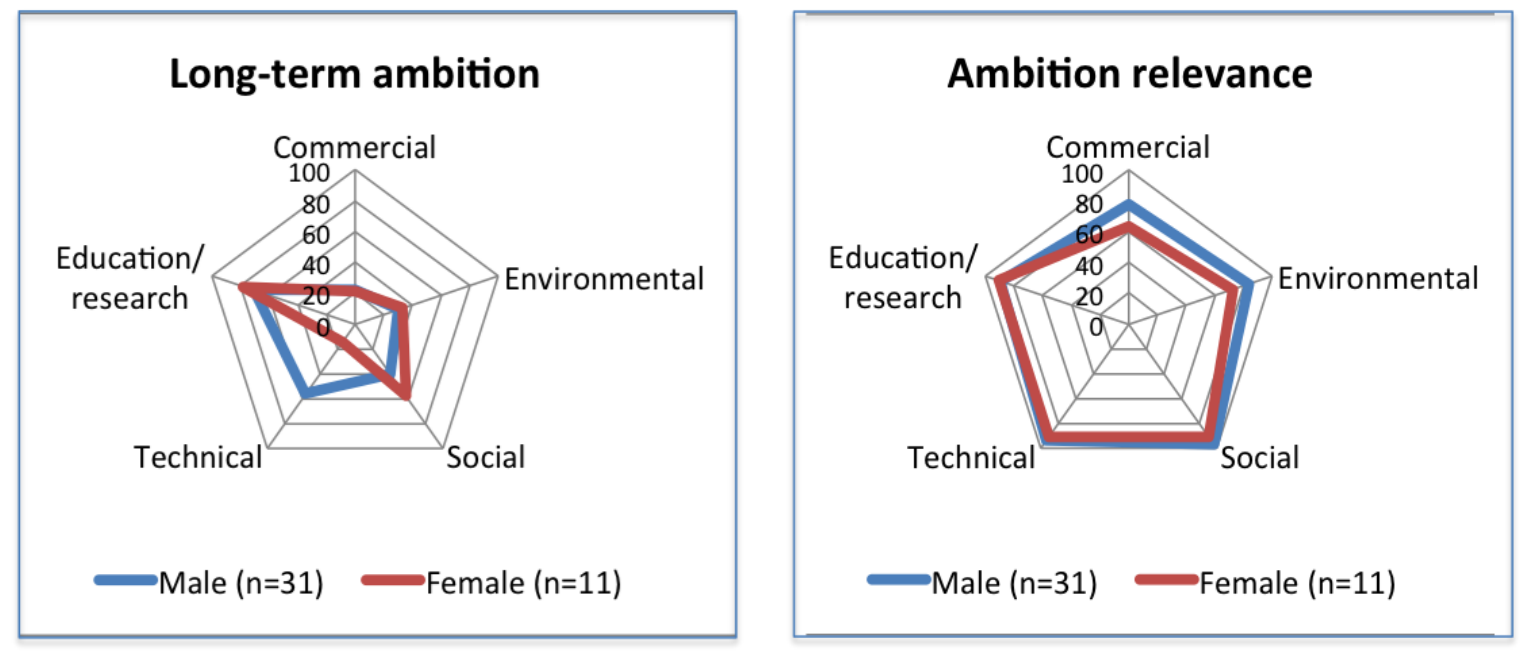

Figure 9. Long-term ambitions and relevance of maker initiatives, by gender. 
The five types of long-term ambitions of the maker initiatives shown in Figure 9 can be compared with these makers' actual reported impact achievements, discussed below. Both educational science and research and technical ambitions seem overall to be the most important, followed by social, environmental and finally commercial. Again, this tends to corroborate the conclusions of MAKE-IT's in-depth qualitative case study analyses of ten maker initiatives. Gender differences are also plotted in Figure 9, which shows quite similar ambition and relevance scores, except that males are much more focused on technical ambitions than females, whilst the latter are a little more focused on social ambitions than males.

Figure 10 shows how the five different scales have different ambitions and the extent to which the initiatives see these ambitions as relevant or not. Ecosystem configurations, followed closely by networks, normally score highest on both. Even though ecosystems are not at the largest potential scale, they can be thought of as exhibiting the most intricate, comprehensive and developed set of relationships between different, but complementary, actors at a relatively large scale. Given this level of development, when successful they are thus likely to be very ambitious and to see all ambitions as highly relevant. The same argument holds, though to a slightly lesser extent, for networks, which tend to be less developed than ecosystems but at a larger scale.
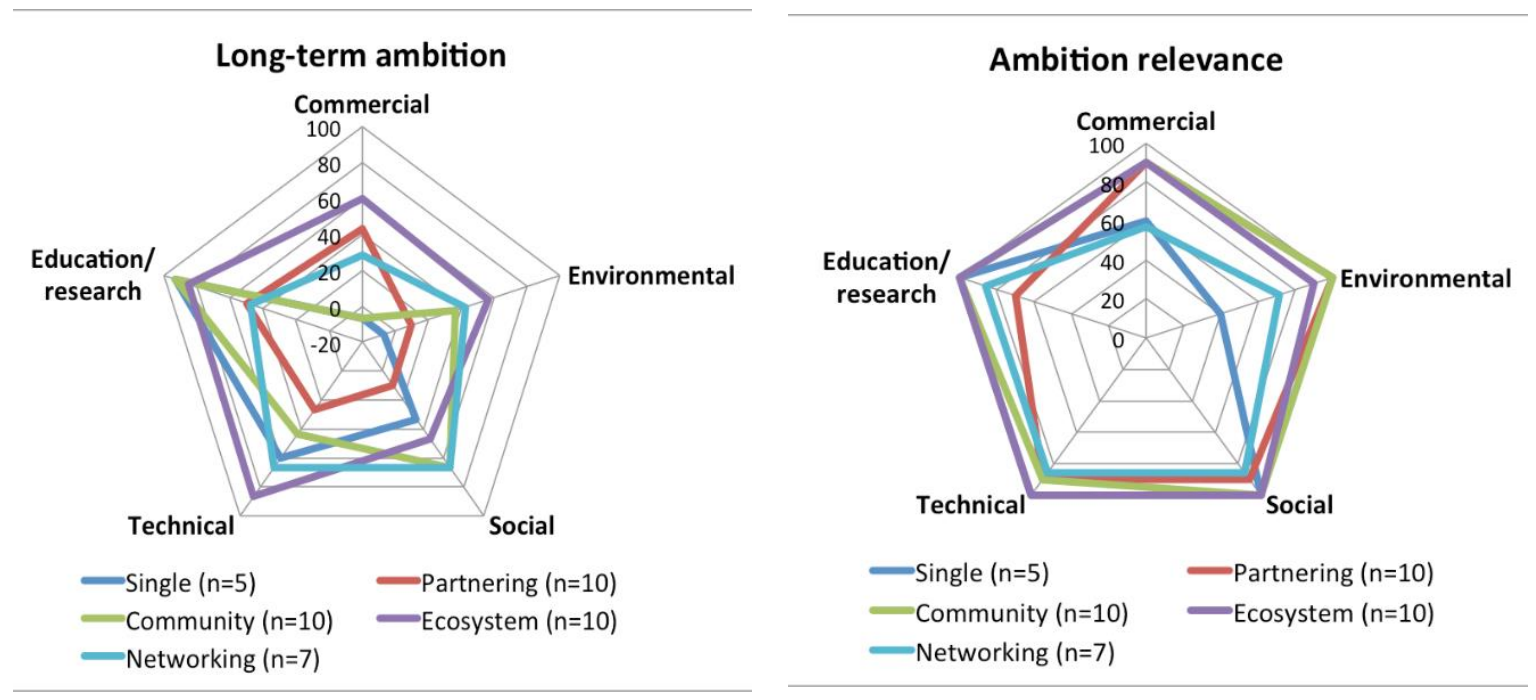

Figure 10. Long-term ambitions and relevance of maker initiatives by scale.

It is also interesting in Figure 10 that there seems to be quite a strong positive correlation between increasing scale and increasing ambition/relevance, with only a relatively minor deviation between the two smallest scales of single and partnering. This observation will be repeated on numerous occasions below when looking at actual achievements. It is quite clear that, like gender, scale is a very important determinant of the success and impacts maker initiatives are likely to have.

\subsection{Organization and Governance}

The overall reported achievements in organization and governance (pillar 1), as shown in Figure 11, are mainly good, although overall the achievement rate is only $33 \%$ of those for whom such achievements are relevant, with some interesting differences between the various issues. Openness to and sharing with partners outside the maker initiative itself is the most positive result, perhaps reflecting the conclusion noted in the discussion of maker scales (Figure 4), where the partnering, community and ecosystem scales are the most common operational forms.

As the case study interviews with makers show, they are very keen on realizing openness as much as possible: "Openness and sharing are the key principles that makers abide by. I haven't seen makers being overly protective of their (intellectual property) IP. They are mostly willing to share 
their materials, designs with anyone who is interested" (maker, Estonia). Openness is practiced by the sharing of ideas and designs locally, and by learning online in the wider community using multiple platforms for uploading designs and projects for other makers to use and adapt. However, for makers with commercial ambitions it is challenging to reconcile the value of openness and protection when running a maker business: "We're of course in competition with other people. We're entering into a capitalist market where money is being made. And if we go there now and document every bit that we make and put it online, then we shoot (ourselves) in our own foot of course" (maker, Germany). Thus, openness challenges the business model of makers.

Financial and organizational sustainability is much less positive in Figure 11, although is still generally achieving some good results. By contrast, however, the maker initiatives' gender balance is reported to be relatively poor, even disregarding those initiatives for which this is not a relevant goal. Overall, the more bottom-up, informal aspects seem to be more important than the more top-down and formal aspects, even in the organization and governance pillar, again indicative of the early stage of maker development. There are also large gaps between achievements and relevance on all issues except gender (where both are low) and openness and sharing (where both are relatively high). Achieving the long-term vision shows the largest gap, which is perhaps unsurprising given the relatively early stage of development of these maker initiatives.
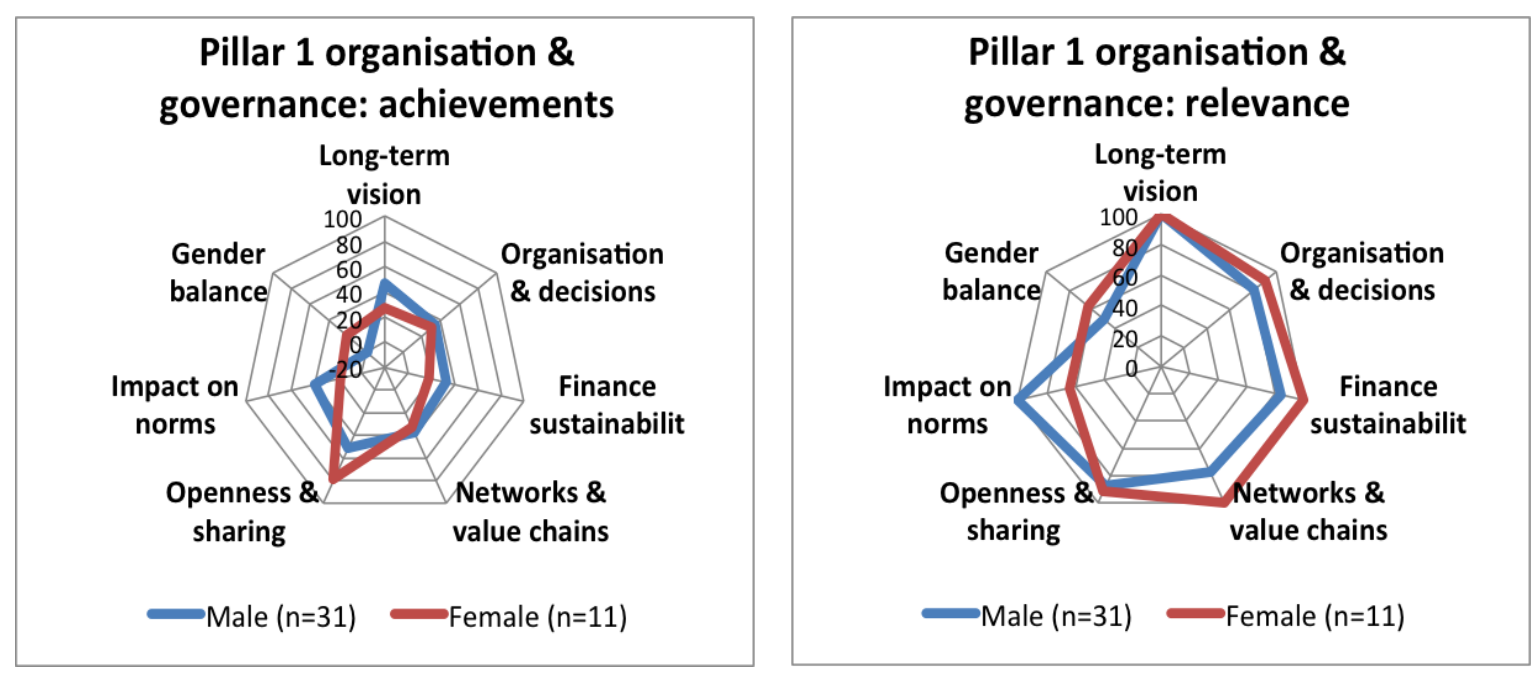

Figure 11. Organization and governance achievements and relevance: by gender.

In terms of gender contrasts, Figure 11 clearly shows that females have both greater achievements and greater relevance on gender balance compared to males, although this remains on the low side. Females also have greater achievements on openness and sharing, whilst males feel they are achieving a little more on changing prevailing norms, policies and regulations, on their long-term visions and on financial sustainability.

When examining the five types of scale in Figure 12, again the two largest scales, networking and ecosystems, both report the biggest achievements.

However, in the organization and governance pillar, networking is leading ecosystems, perhaps because it is more concerned with the larger scale external environment compared to the peer and collaborative behavior pillar, where ecosystems are more successful than networks. There are also large gaps between actual achievements and relevance across all scales, although the largest scales have the smallest gaps given that they are generally more successful. The gaps are most marked in relation to gender balance but also in terms of organization and decision-making as well as financial sustainability. Again, there is a very strong positive correlation between increasing scale and increasing achievement/relevance. 

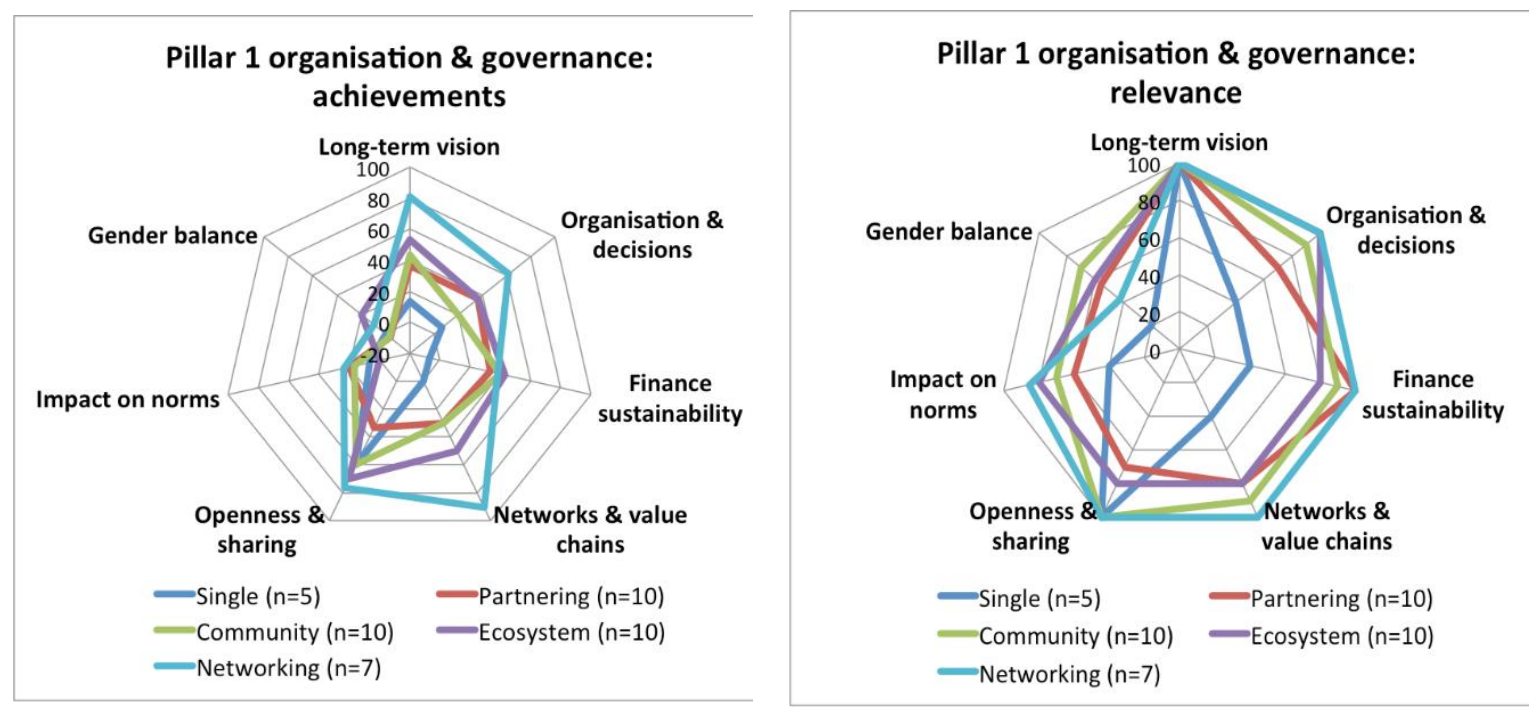

Figure 12. Organization and governance achievements and relevance: by scale.

\subsection{Peer and Collaborative Behaviours}

The overall reported achievements in peer and collaborative behaviors (pillar 2), as shown in Figure 13, are generally very positive, and overall are greater than in the organization and governance pillar, with an average achievement rate of $40 \%$. This may be because many makers are still at a relatively informal stage, experimenting and playing with the technology and with co-creative, collaborative and self- and group-learning activities, so this result perhaps reflects this early stage of maker development. The result may, however, be somewhat biased because, as noted above, many of the individuals interviewed in the sample were lead practitioners rather than management or administrative staff.
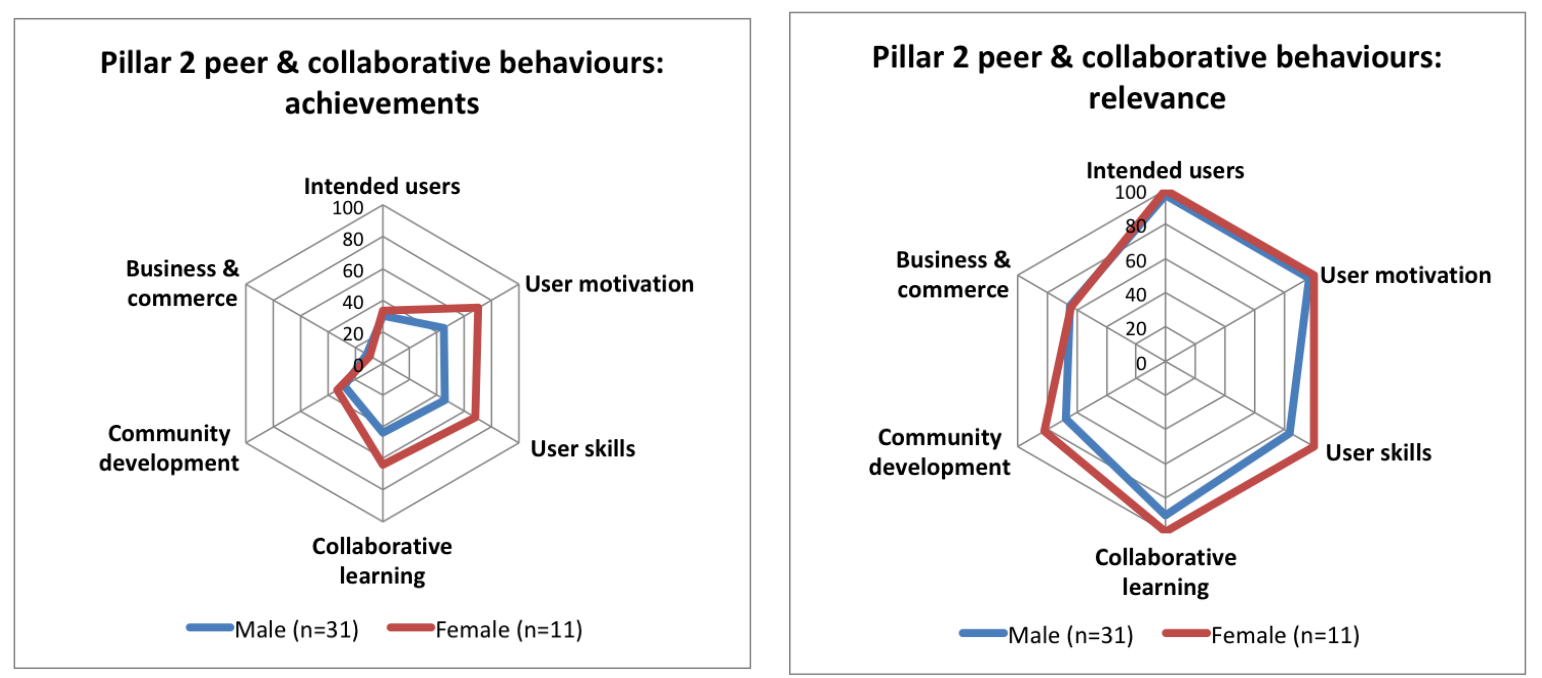

Figure 13. Peer and collaborative behavior achievements and relevance: by gender.

The most positive results in Figure 13, come from questions on the motivation and engagement of users, collaborative learning and knowledge, and individual user skills and competences, in each of which there is also high relevance across most of the initiatives surveyed and with very few negative or neutral reports. This probably reflects the conclusion above that these types of achievement reflect the current preoccupation and focus of most makers. In contrast, achievements related to intended 
business and commercial goals are much more evenly spread between both positive and negative results from the questionnaires, resulting in lower overall scores, in addition, one third of the maker initiatives surveyed do not even have such goals so their relevance is relatively low.

In terms of gender contrasts, Figure 13 clearly shows both that females have higher aspirations (given by their relevance scores) and are in practice achieving much more on user motivation, user skills and collaborative learning, compared to males. This conclusion, i.e., that female-led maker initiatives are achieving more, and are thus generally more successful, than male-led initiatives, is further reinforced in much of the data presented below.

In relation to scale effects, Figure 14 again shows the predominance of the two largest, with ecosystems claiming the biggest achievements and a generally very clear positive correlation between increasing scale and greater achievements/relevance. The largest gaps between achievement and relevance are for business and commerce.

The case studies show that the level of engagement of makers depends on their willingness to participate in regular activities and their unwillingness to invest in high cost projects. The lack of trust that inhibits access to some maker spaces was mentioned as a barrier to deeper involvement. However, makers stated they were driven by open access to equipment and knowledgeable professionals. The more the makers were able to test and use these services and tools, the more they become engaged in making. Thus, it seems that maker engagement is mainly dependent on their personal characteristics, e.g., high motivation and interest, in the context of access and opportunity.
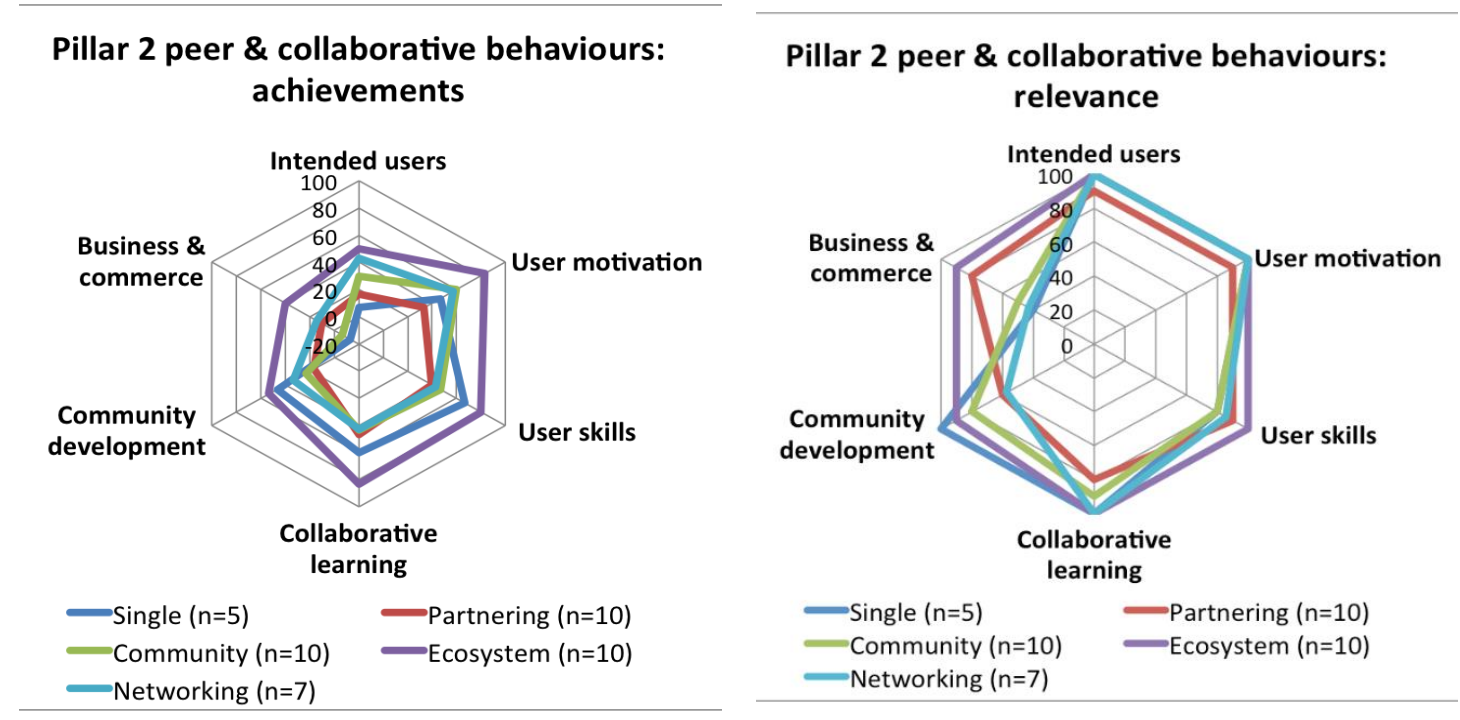

Figure 14. Peer and collaborative behavior achievements and relevance: by scale.

\subsection{Social Sustainability Achievements}

As shown in Figure 15 achievements related to social sustainability are generally good, although overall the achievement rate is only $29 \%$ of those for whom such achievements are relevant. Improved human capital appears overall to be the most positive, probably related to the questions on collaborative learning and knowledge, and on individual user skills and competences, referred to in the peer and collaborative behavior pillar above.

Improved education and research achieves the second highest impact in Figure 15. The questions on changed social opinions and behavior, improved quality of life and on improved social inclusion and cohesion are less positive but still overall more positive than negative. These latter three questions are ostensibly broader and perhaps less concrete than improved human capital, that can be related more or less directly to measurable skills, so there is likely to be both more uncertainty in scoring them higher as well as greater difficulty in assessing such impacts in the wider community. 

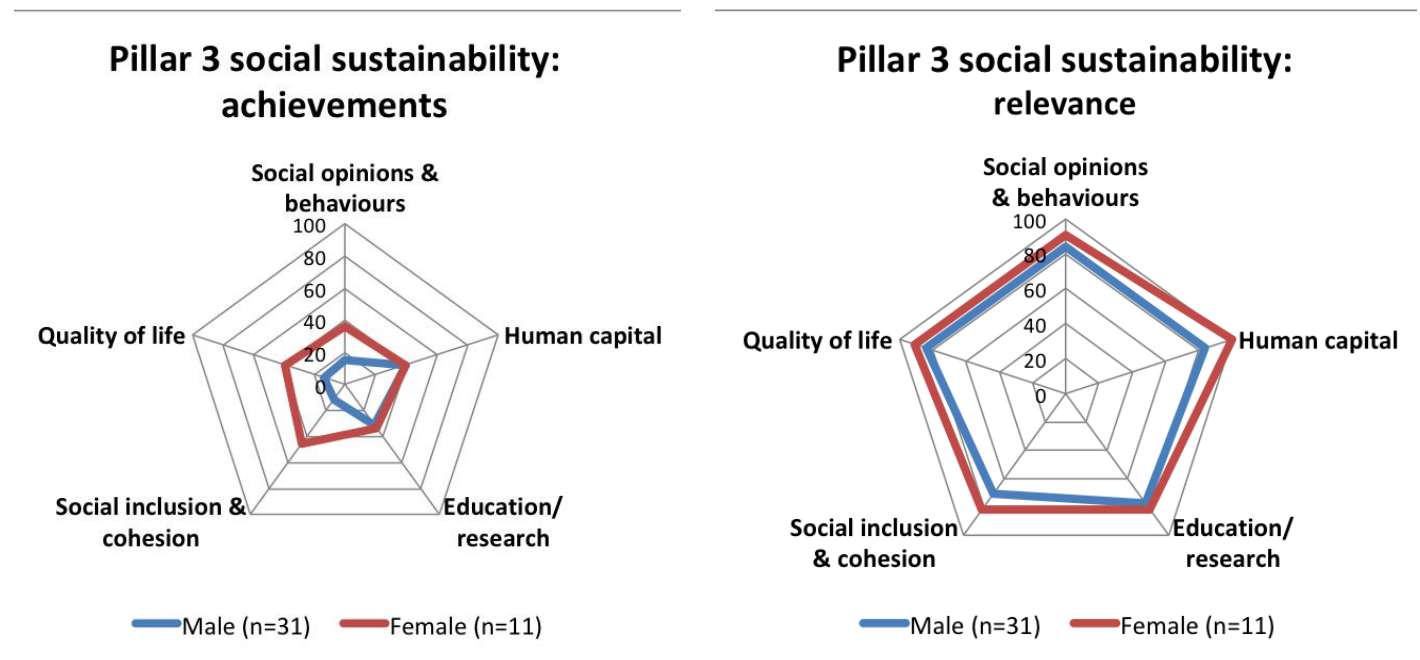

Figure 15. Social sustainability achievements and relevance: by gender.

Looking at gender issues in Figure 15, female-led initiatives are having much greater success in achieving social sustainability, particularly in relation to social inclusion and cohesion, quality of life and social opinions and behaviors. In fact these would score quite high overall except for the fact that male-led initiatives seem to perform badly. However, both genders perform relatively well on human capital and education and research which, as mentioned above, can be more readily related to measurable outcomes such as skills.

Turning to scale differences for social sustainability, Figure 16 shows that ecosystems score, very decisively, much better than all others across all five issues. The other scales tend to be more random in their achievements, with networks scoring best on social inclusion and cohesion, communities on human capital and quality of life, and singles on education and research. These all appear quite rational, as does simple partnering which seems to score lower than all others across most issues given that partnerships tend to be focused in their activities, as noted above in relation to their commercial activities. Ecosystems also score highest in terms of perceived relevance, with overall generally a positive correlation between increased relevance and increased scale.
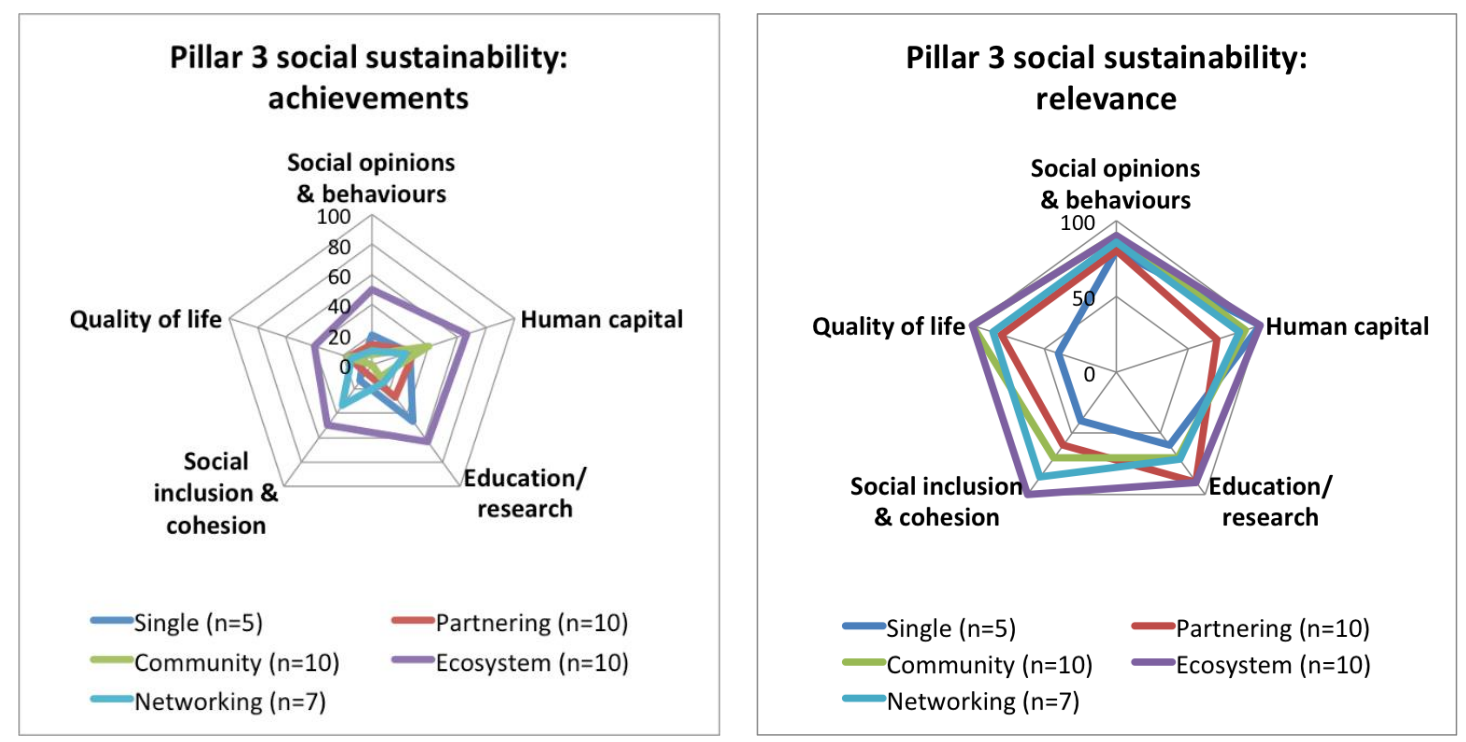

Figure 16. Social sustainability achievements and relevance: by scale. 
The case studies attest all these social sustainability achievements, especially in terms of education, training and innovations addressing social challenges. Education is described as the core aim of maker spaces, with all cases offering workshops for educational institutions, from kindergarten children up to university students: "( . . ) Part of the task, which we set ourselves is of course to try to break barriers, especially for pupils who would never get the idea to study because they grow up in a social environment where they have no contact at all to universities. ( ... ) social origin determines the educational career a lot here" (manager, Germany). Thus, making might have an impact on career choices as well as an empowering effect: "Something that is very remarkable is the fact that whatever happens in our lab, our advisory service is all about the empowerment of people, providing people with knowledge and tools that make them become more valuable, whatever they do. It is giving them knowledge, practical tools and approaches that strengthen their capabilities" (manager, Denmark). There are also numerous examples of maker innovations that tackle social challenges and enhance quality of life, from printed prostheses for people with disabilities, to mobile maker spaces in disaster zones for developing products to tackle immediate necessities (Fieldready.org).

\subsection{Economic Sustainability Achievements}

Economic sustainability achievements, shown in Figure 17, are generally as high as social sustainability achievements with an achievement rate of $28 \%$, but they have less impact overall because the percentage of initiatives for which these questions are relevant is $71 \%$ compared with $83 \%$ for the social sustainability questions. This leads to a tentative conclusion that for those fewer initiatives that have economic sustainability goals, they are achieving them relatively well, whereas there are more initiatives with social sustainability goals and all these are also achieving them relatively well. There are also interesting differences between each of the economic questions, as shown in Figure 17, with the improved sharing, collaboration and co-creation in an economic context question the most positive. This probably relates, as noted above, to the highly positive impact questions on collaborative learning and knowledge, and on individual user skills and competences, referred to in the peer and collaborative behavior pillar above.
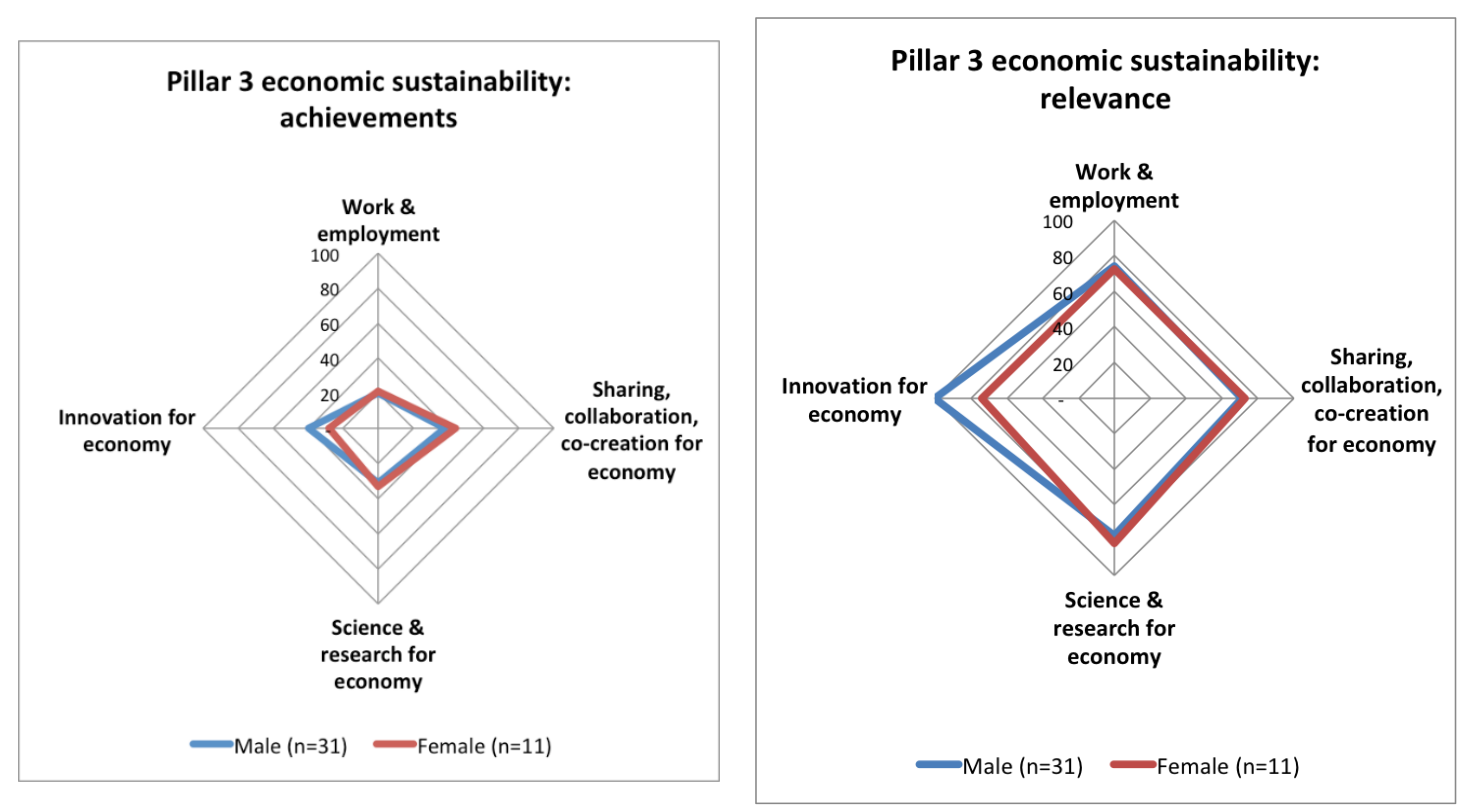

Figure 17. Economic sustainability achievements and relevance: by gender.

Also important are questions on improved science and research for the economy and improved innovation in the economy, showing the general value of maker initiatives to these issues. Performing 
least well are work and employment, although, it needs to be stressed, some achievements are being made in this context. As concluded earlier, the improved sharing, collaboration and co-creation question directly reflects the main activities in the current stage of maker development, i.e., focused somewhat more on experimenting and playing with the technology and with co-creative, collaborative and self- and group-learning activities. The other questions reflect impacts that probably require a longer time horizon and changes to more established institutional structures and activities.

There are only minor gender differences shown in Figure 17, with the most prominent being greater male economic sustainability achievements in innovation for the economy, but slightly less than females on sharing, collaboration and co-creation.

In examining scale differences, Figure 18 shows a very strong positive correlation between increasing scale and increasing sustainability achievements across all four issues, again with the usual flip between ecosystems and networks. Thus, both in terms of social and economic sustainability achievements, ecosystems are clearly the most successful scale, followed by networks and then the smaller scales progressively performing less well. For both gender and scale, relevance scores closely mirror achievement scores with quite large gaps across all four issues.

The case studies show that economic sustainability is mainly generated through easy prototyping and the drive for innovation, as well as impacts on the labour force. Using digital fabrication tools, prototypes can be easily developed and ideas tested before needing substantial funding: "I think they [FabLabs] are a kind of nucleus for products in general, because you can develop your ideas with no risk. If you have an idea, you just come here and try it out. If you figure out that it did not work, then it just does not work, but you did not buy a laser cutter for $€ 30,000$ before trying. No start-up business could afford that" (maker, Austria). Thus, products find their way to the market faster and ideas can be tested before searching for investment, showing that making is a driving force for innovation. Indeed, many start-up companies have their roots in the maker movement, whilst impact on the labour force comes from local job creation and up-skilling the work force.
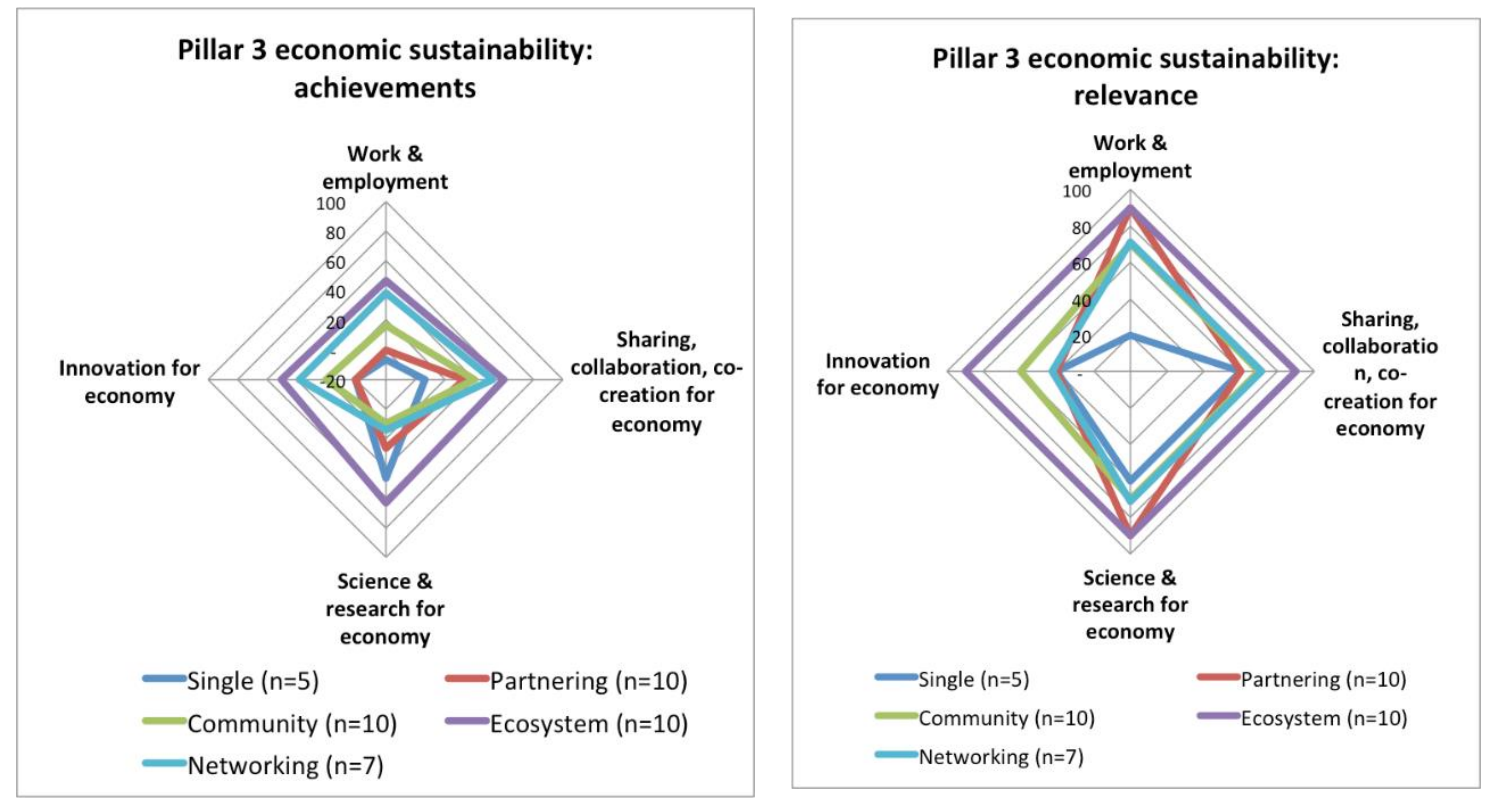

Figure 18. Economic sustainability achievements and relevance: by scale.

\subsection{Environmental Sustainability Achievements}

Compared with both social and economic sustainability achievements, environmental sustainability achievements are overall somewhat less impressive, with an achievement rate of only $15 \%$, indicating both the overall lack of ambition in this regard as well perhaps the difficulty for 
makers in undertaking environmental evaluations. However, there are some quite marked differences between individual environmental questions, as revealed in Figure 19.
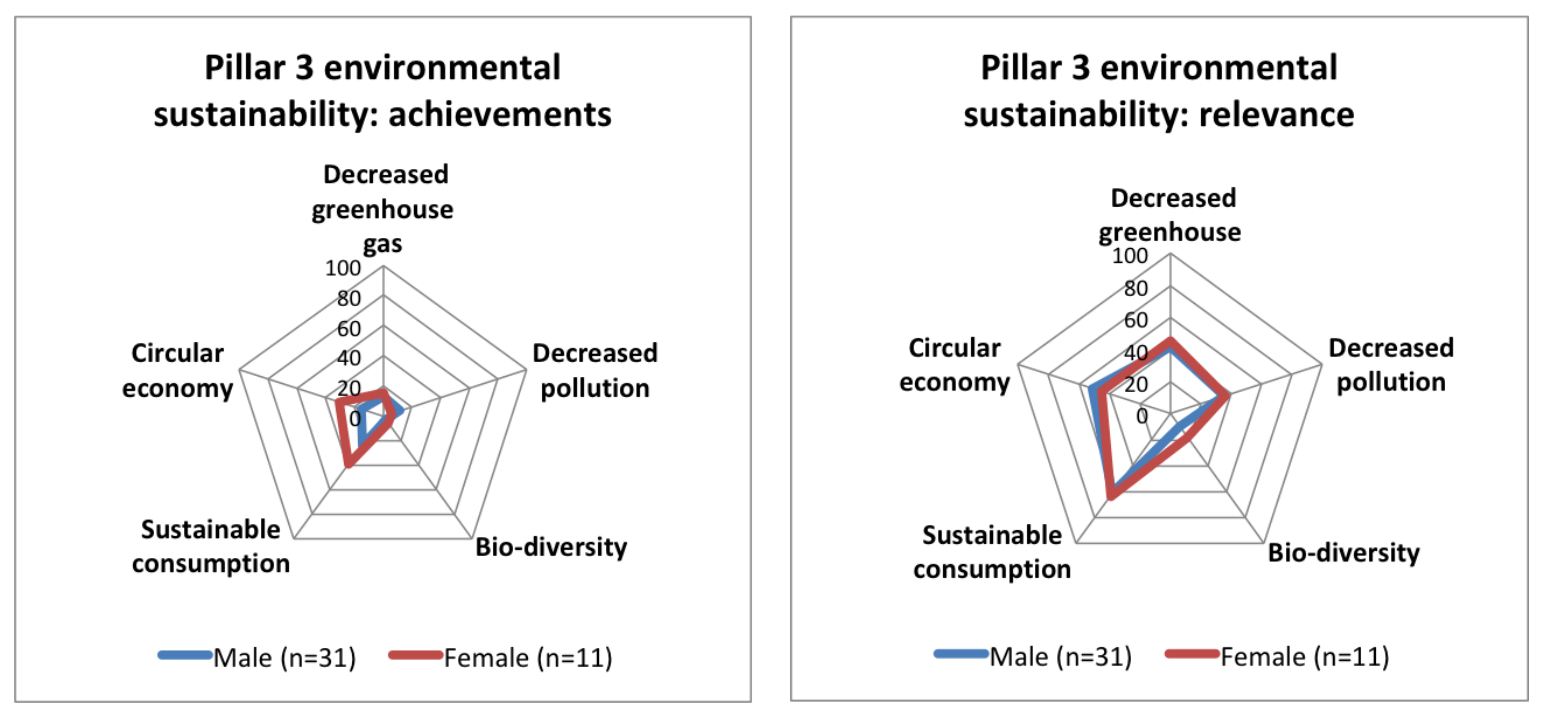

Figure 19. Environmental sustainability achievements and relevance: by gender.

More sustainable consumption shows the greatest success in Figure 19, perhaps related to the strong material re-use and high resource use efficiency of makers. Although collectively sustainability achievements are lower than overall social and economic achievements, the specific issue of sustainable consumption is achieving almost as high sustainability impacts as the highest social and economic issues. Circular economy sustainability impacts are also relatively good although less than sustainable consumption, even though the two relate, probably because the former are more likely to be part of broader industrial systems into which only few makers are currently incorporated. In contrast to these two, sustainability achievements related to decreased greenhouse gas emissions and of other types of pollution, as well as of improved bio-diversity, are only marginally positive, perhaps indicating the difficulties for makers in understanding in practice how they might contribute directly to these goals.

These results perhaps reveal a weakness in the methodology here, as there are two issues which perform well and on a par with many social and economic impacts, but these are coupled with three which perform much less well, thereby significantly reducing the average achievement of environmental sustainability. Although decisions about the issues to address have been derived from desk research and widespread consultation with makers, the actual choice can obviously affect the average result. Clearly, there are some quite good environmental sustainability achievements arising from maker initiatives shown by these results.

Distinctions in environmental sustainability achievements related to gender are also seen in Figure 19 with females being clearly more successful than males in relation to sustainable consumption and the circular economy, and marginally less successful in terms of decreased pollution, the latter perhaps being a more technically specialist issue.

Looking at scale differences, Figure 20 shows that there is again, in general terms, a clear correlation between increasing scale and impact. However, in relation to environmental as opposed to social and economic sustainability achievements, networks do perform better than ecosystems, but also partnerships perform better than communities. This seems to reinforce the evidence above that networks and partnerships are both more focused on, and more specialist in, more challenging technical and commercial issues. Environmental challenges are perhaps a good example of this, being able to exploit either small strong ties as in partnerships, as well as perhaps looser although very wide -ranging ties as with networks. It is also noteworthy that singles do not appear in Figure 20 because 
none of the five interviewed could answer the environmental questions, unlike all interviewees at other scales and unlike all the other questions that they did respond to.
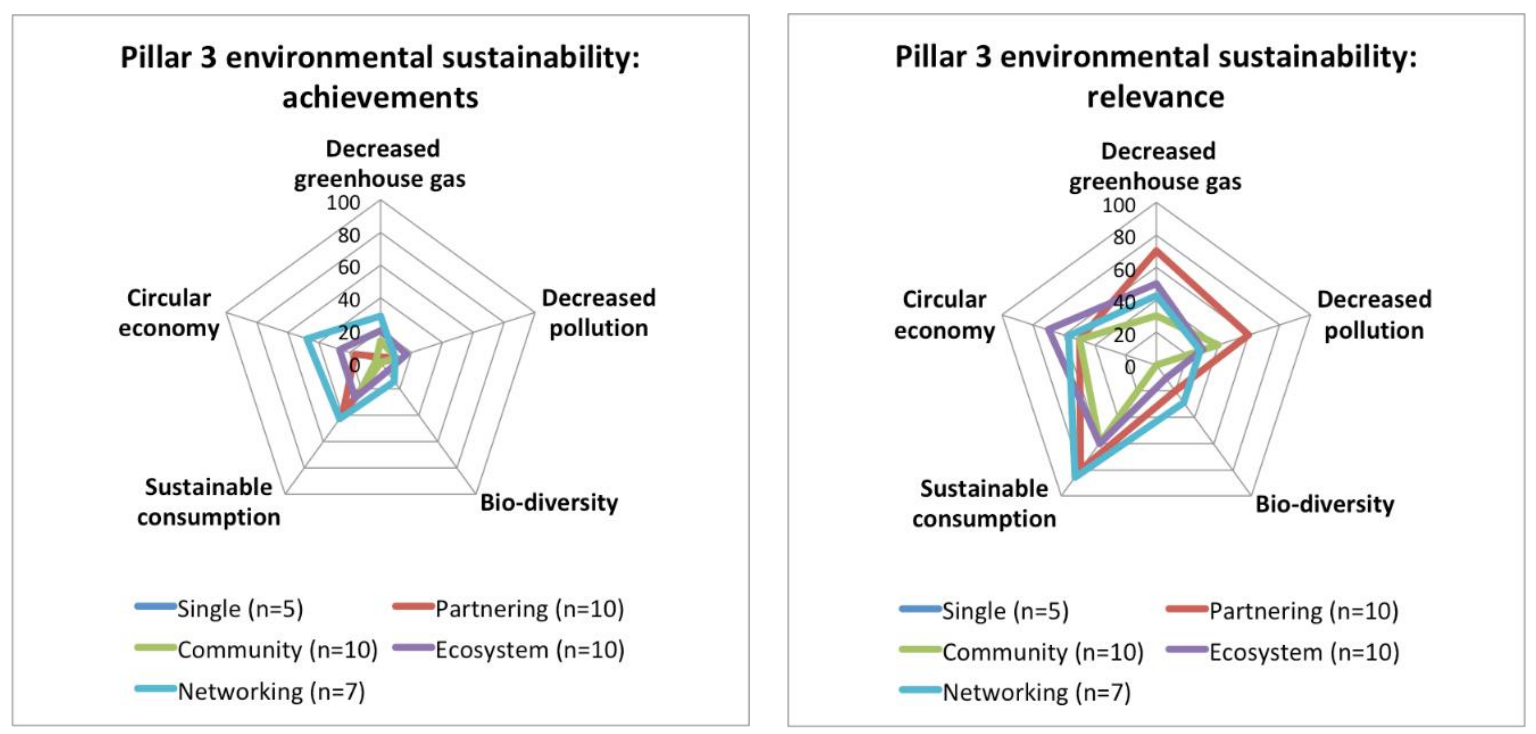

Figure 20. Environmental sustainability achievements and relevance: by scale.

Some of the case studies addressed environmental challenges with maker inventions (e.g., a water purification tool using solar energy, an energy-harvesting flower-pot, etc.), others experimented with environmentally friendly materials (e.g., mushroom-based textiles replacing leather) or developed substitute parts. While not all makers in the cases were environmentally conscious, there were many examples of makers keen on up-cycling, re-cycling or repairing things in their maker space [19].

\section{Discussion}

There are a number of general discussion points arising from the results and analysis reported above, both from the 42 maker initiatives investigated in Barcelona, and from the ten case studies drawn from across Europe, most of which appear to be complementary. These are grouped under four headings: technology; ambitions and achievements by analytical pillar; the importance of gender; and the importance of scale. In addition, links are drawn to some wider issues of sustainability and to the ongoing development of the maker movement in this context.

\subsection{Technology}

The technology types used cover the whole range: 3D printing, modeling, robotics, Internet of Things (IoT), software development, telecommunications and electronics. It is unsurprising that 3D printing technologies are the most common, given that these are, by and large, what defines who makers are. However, a large number of other technologies are also used, by far the majority of which are not specifically offered to or designed for makers. In terms of the activities for which the technology is used, there is strong emphasis on sharing, collaboration, learning and interaction: These are more typical of the peer and collaborative behaviors pillar, whilst the least used categories are organization and communication that are more indicative of organization and governance pillar activities. This shows that makers are generally still at a quite informal, less organised stage, more concerned with starting up and exploring possibilities, which the peer and collaborative behaviors pillar tends to represent. 


\subsection{Ambitions and Achievements}

In terms of the ambitions of maker initiatives, education and research are the most important, followed by social and technical. Of less importance are environmental ambitions and the least important are commercial ambitions. These priorities tend to reflect some of the main reasons for starting a maker initiative, i.e., to provide skills upgrading for both vocational and non-vocational purposes often in educational institutions, and to serve social and sometimes inclusion goals. Technical ambitions are also an intrinsic part of this given the new and innovative nature of the technologies being used which tend to attract young people, especially males, to explore the new possibilities.

Similar to ambitions, the achievements actually being delivered are generally higher for the peer and collaborative behaviors pillar than the organization and governance pillar, again perhaps underlining the still relatively early stage of development of many maker initiatives. This result is further underpinned by the fact that the overall focus of achievements tends to be on the more bottom-up and sometimes informal individual aspects of the peer and collaborative behaviors pillar (like user skills and motivation, sharing and learning) than on the more top-down and formal organizational aspects (like management, decision-making, impact on wider institutional norms, including policies and regulation) of the organization and governance pillar.

Looking at the social sustainability impacts achieved, improved human capital appears to be the most positive in the social context, probably related to the questions on collaborative learning and knowledge, and on individual user skills and competences, referred to in the peer and collaborative behaviors pillar above. In terms of economic sustainability impacts, improved sharing, collaboration and co-creation in an economic context are the most positive. Also important are questions on improved science and research for the economy and improved innovation in the economy. Fewer impacts are, however, currently being achieved in relation to improved work and employment, perhaps because these tend to require achievements over the longer term.

The most important environmental sustainability impacts are more sustainable consumption and circular economy impacts, perhaps related to the strong material re-use and high resource use efficiency of makers. In contrast to these two, impacts related to decreased greenhouse gas emissions and of other types of pollution, as well as of improved bio-diversity, are only marginally positive, perhaps indicating the difficulties in understanding in practice how makers can contribute directly to these goals.

In general for the overall societal sustainability impacts pillar, there are both good social and economic sustainability achievements, but there are many more of the former that are rated as relevant, so the overall social sustainability impact is greater. Environmental sustainability impacts are collectively rated as less relevant, perhaps because they are more difficult to understand and engage with, although there are some very good positive environmental sustainability results.

\subsection{The Importance of Gender}

Most maker initiatives are led by young males which is quite similar to the situation seen in the majority of technology-based start-ups and new enterprises. However, as summarized below, females generally achieve better results than males. In terms of technology use, males and females generally use the same technologies to a similar extent. However, females tend to use a wider range of technologies and to be less specialized in their technology use. Females are also more likely to use more interactive and collaborative tools and be leading maker initiatives at larger scales, i.e., networking and ecosystems, than males (see below), where such technology uses are most relevant. By contrast, males are marginally more likely to be using the technology for commercial purposes, and to be much more focused on technical ambitions than females, whilst the latter are somewhat more prone to have social ambitions than males.

In relation to the organization and governance pillar, females report both greater achievements and greater relevance on gender balance compared to males, though this remains on the low side for both. Females also report greater achievements on openness and sharing, whilst males feel they 
are achieving a little more on changing prevailing norms, policies and regulations, on their long-term visions and on financial sustainability. In terms of the peer and collaborative behavior pillar, females report much higher aspirations (given by their relevance scores), and are in practice achieving much more on user motivation, user skills and collaborative learning, compared to males. Overall, females have a higher focus on social and educational issues, on openness and sharing, skills and quality of life, whilst males focus more on technology, industry/economy innovation and changing regulations, norms, etc.

Female-led initiatives are having much greater success than males in delivering social sustainability impacts, particularly in relation to social inclusion and cohesion, quality of life and changing social opinions and behaviors. On the other hand, there are greater male achievements in economic sustainability related industry and economy innovation, but slightly less than females on sharing, collaboration and co-creation in an economic context. In the environmental sustainability impact context, females are also more successful than males in relation to sustainable consumption and the circular economy. However, females are marginally less successful than males in terms of decreased pollution, the latter perhaps being a more technically specialist issue.

The maker initiatives that females and males lead seem to be operating at quite different scales. Females are much more likely to work at the larger scales of ecosystems and networks, although they are also well represented at the community and less so at single scales. Males, in contrast, are much more likely to quickly move from the single stage to partnering, which seems to be their dominant modus operandi, although they are also well represented in communities and less so in ecosystems.

The overall conclusion is that female-led maker initiatives are achieving more, and are thus generally more successful, than male-led initiatives. The reasons for this are difficult to unravel, especially as expectations would point to the opposite in such a male dominated context. Clearly many females can bring unique skills and insights which complement male ones. However, a contributing factor might be that only high caliber females lead maker initiatives, precisely because of the barriers against them, whilst the range of male leaders is much wider so their average level of skill, determination and ambition may be lower, leading to fewer achievements overall. Another reason might be that females have to be more ambitious and expert from the outset in this male-dominated field. Whatever the reasons, females are in most cases underrepresented in most maker initiatives. To change this, there is a need to actively promote a culture that promotes diversity (not only females, but also other groups which tend to be left out, such as the elderly) and to install a respectful and supportive culture in maker spaces [17].

\subsection{The Importance of Scale}

Looking at each of the five scales in turn (see Figure 4), single/individual initiatives seem to be quite diverse in their technology use, their ambitions and achievements, compared to the four other scales each of which do seem to demonstrate distinct characteristics. Perhaps this is the case given that singles can be highly diverse, but possibly also because their sample size for this analysis is the smallest at only five initiatives. Maker initiatives characterized as partnering tend to use 3D printing and modeling technologies more than other scales, and electronics, robotics and IoT less. Partnerships are also more likely to be more focused and specialized in their activities, especially in relation to commercial and environmental ambitions and achievements, compared to the larger scales of community, ecosystems and networks. Partnering is thus perhaps better able to provide the close bonding and trusting relationships necessary for makers to set up good commercial relationships in the maker context than are other scales. By contrast to partnering, communities tend, as suspected, to consist mainly of single, though sometimes several, maker initiatives having very close relationships with their users. These relationships are mainly non-commercial, and the overall ambitions and achievements of communities are also more focused on social sustainability rather than economic sustainability impacts. 
Moving to ecosystems and networks, these larger scale initiatives seem almost always to achieve much bigger impacts than smaller scale initiatives. However, ecosystems outperform networks in terms of the peer and collaborative behavior pillar as well as in social sustainability and economic sustainability, perhaps because they consist of well functioning local or regional clusters of complementary and diverse actors. Networks, however, outperform ecosystems in terms of the organization and governance pillar and environmental sustainability, possibly because they sometimes have an even large scale and much learning between similar initiatives given the need to challenge many more incumbents, infrastructures and norms than the peer and collaborative behavior pillar, or social and economic sustainability, at least in the early stages of maker development. Even though ecosystems are not necessarily at the largest potential scale, they can be thought of as exhibiting the most intricate, comprehensive and developed set of relationships between different, but complementary, actors at a relatively large scale. Such relationships are typically at the heart of an innovative milieu. When successful, ecosystems are therefore likely to be very ambitious and to see all ambitions as highly relevant.

Many of the observations concerning ecosystems also hold, though to a slightly lesser extent, for networks, which are likely to be less developed than ecosystems but at a larger scale. It is also relevant to note that the two largest scales of ecosystems and networks tend to be more "all-round" in their use of technology, which again is perhaps not surprising as they involve many more actors and are generally more complex and comprehensive in the range of their activities.

The overall conclusion regarding the scale of maker initiatives is that the scale differentiation proposed in Figure 4 is strongly supported empirically, and that large scales almost always do better in terms of ambition, achievement and relevance than smaller scales. The largest scales also have the smallest gaps between achievements and ambitions given they are generally more successful. Scales thus provide a robust and empirically valid framework for analysis.

Thus, to maximise the successful operation and impacts of maker initiatives, it is clear that ecosystems and networks, both formal and informal, should be supported by policymakers and strategies at different levels and sectors. This does not mean that the smaller scales are not important nor valuable, as they provide good routes into making and can achieve much on their own, but encouraging and linking them towards greater and larger scale awareness and cooperation can provide win-wins all round.

\subsection{Development of the Maker Movement}

As highlighted in this discussion, the current status of maker development seems still to be at an early stage with a lot of enthusiastic and innovative activity. They are just starting to make significant progress on more formal internal organization and management and becoming embedded in wider governance and institutional contexts, whilst having important, though not yet large scale, social, economic and environmental sustainability impacts on wider society. Given this, it is important that policy focuses on nurturing the maker movement to help it better achieve its potential across the wide spectrum of social, cultural, economic and environmental sustainability benefits it can clearly deliver. The increasingly important links with mainstream industry, as much of this moves towards distributed manufacturing business models, is also extremely important.

It is also quite clear from the discussion above that, like gender, scale is a very important determinant of the success and sustainability impacts maker initiatives are likely to have. The maker movement started in garages, basements and workshops, set up "maker-spaces", joined and often became indistinguishable from "hacker-spaces" and formed communities on the web. They have become platforms for learning, sharing and collaboration. Many observers thus point to the social sustainability of making, increasing the social cohesion, inclusion, wellbeing, and quality of life of those it engages. They see its potential to change behaviors towards more sustainable lifestyles. At the same time, they see possibilities for transformations in economic sustainability through new skills and jobs, innovations in products, materials and business models, for example where digital 
designs for physical products are shared and then used or extended by third parties. This culture of experimentation is a powerful driver for innovations leading to social, economic and environmental sustainability $[1,2,7,11,20]$. Typically, products go through several design iterations reusing local material feedstocks, each adding new features which better suit specific needs as makers increase their understanding of the communities they work with. For example, a basic water purifier using solar energy that can be adapted with reusable parts made of biologically degradable materials to cater for wear and tear $[19,21]$.

The most prominent current manifestation of the maker movement are Fab Labs which have emerged as spaces for democratizing digital fabrication. Here people learn how to design and make with tools and machines that work with the interactions of ICT, physical and biological processes and materials [22]. The maker movement and the Fab Lab network is now being envisaged and extended at city scale and networked globally for the purpose of ensuring that Industry 4.0 will be efficient, effective, as well as socially and environmentally sustainable. According to the Fab City whitepaper [23], cities should rapidly move towards being locally productive and self-sufficient whilst globally connected. (See Figure 21) The 18 members of the global Fab City network are committed to moving from the current linear industrial production model, which imports raw materials and products and exports waste and pollution, towards a spiral innovation ecosystem in which energy and materials flow locally within cities, whilst information and data (including on how things are made) circulate globally.

It is claimed that merging the bottom-up maker movement with distributed manufacturing at a larger scale will result in value and supply chains moving away from centralized mass production towards decentralized distributed manufacturing and mass customization [1-4]. This would profoundly impact the future of manufacturing and our physical world, as well as of work, behavior, local and city development, not to mention global politics. Perhaps of even greater significance will be the potential impact on the environment and sustainability more generally. If in the future the only economically traded commodities over large distances consist of (i) talent (high caliber skilled people), (ii) raw materials and energy inputs in cases where their occurrences are still geographically fixed, and (iii) digital algorithms, this will have a hugely beneficial environmental impact as the number of physical goods transported over even medium distances shrinks. The social, economic and environmentally sustainable potential of making is thus of strategic importance.

There is also clear and important potential for maker initiatives, particularly when linking with larger scale industry, to make significant contributions to promoting and fully exploiting the sustainability benefits of the circular economy. While there are about 114 different definitions of circular economy [24], there is agreement that the term means to reduce, reuse and recycle the resources in the process of production, transportation and consumption. The circular economy is perceived in contrast to the linear and prevailing model of "take, make and dispose" associated with creating huge amounts of waste. According to Ghisellini et al., there are three main implementation levels of the circular economy: the macro city level, the meso eco-industrial parks level, and the micro single company level [25]. In light of the Fab City discussion, to this could perhaps be added a super-macro level above cities, i.e., regionally, nationally and globally across borders. Evidence for this comes from Bragdon [26], as it is clear that, at least at this stage of development, the most successful circular economy examples are multi-national companies like Unilver simply because they cover so many different sectors ranging across countries that they are able to use the "waste" from one sector as an input to a completely different sector. Thus their circular economy maneuverability is much greater. Single city or single sector circular economies, although very important, tend to be somewhat constrained because they have fewer options for inputting their "waste" elsewhere. Maximizing synergies and links between different sectors is clearly the way to go to optimize the benefits of the circular economy.

The maker movement, which as outlined above started bottom-up at a small scale but is now having its greatest sustainability impacts at ever larger scales (described above in terms of ecosystems and networks), is starting to impact the industrial scales through Fab Cities and similar developments. 
Its implications are prominent in the European Commission's Factory 4.0 policy (https: / / ec.europa. eu/digital-single-market/en/blog/factories-40-future-european-manufacturing) as well as in parallel national initiatives in Germany and Denmark [4]. It is also being driven by industry itself (as explained by Olivier Scalabre, a member of The Boston Consulting Group's Industrial Goods practice, focusing on the shift from centralized to distributed manufacturing in multiple sectors, such as railway, energy and utility, consumer electronics, and automotive: https://www.bcg.com/en-nor/about/people/experts/ olivier-scalabre.aspx), and not least by the World Economic Forum's strategic research, analysis and policy proposals [6].

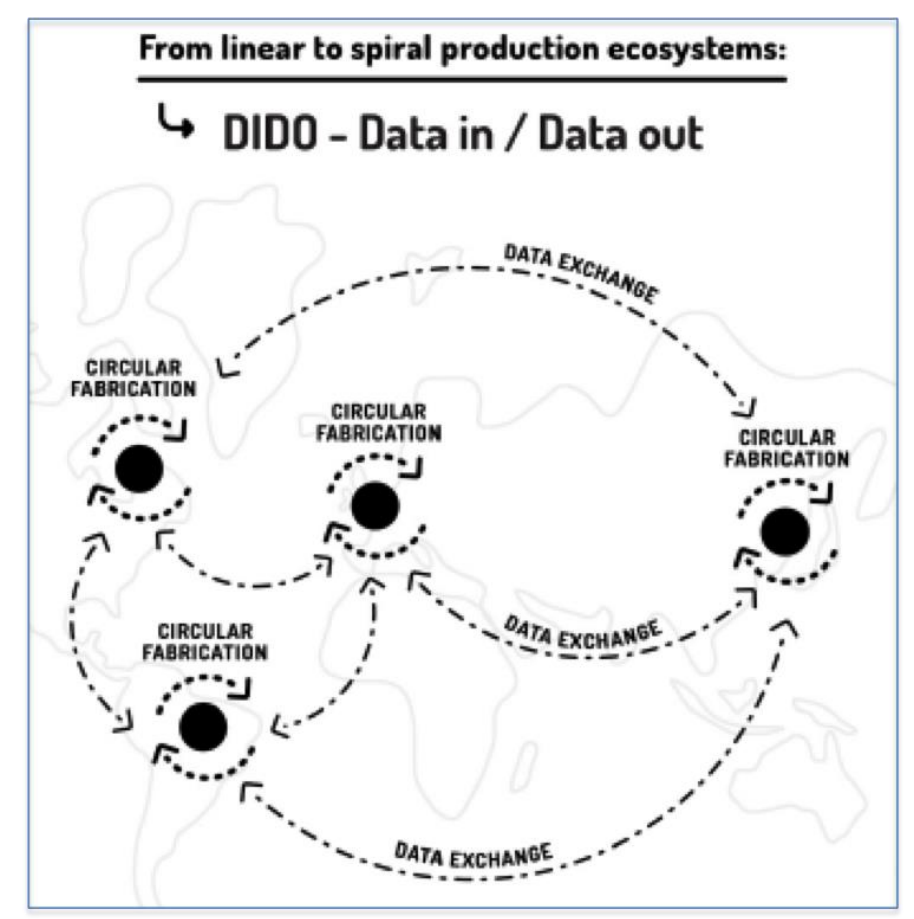

Figure 21. The Fab City concept [23].

\section{Conclusions}

The jury is out on whether the maker movement will be midwife to the next industrial revolution by moving from centralized to decentralized distributed manufacturing, as claimed for example by Anderson [1] and by Olivier Scalabre more recently (See above). The World Economic Forum itself is, however, presenting a range of views. On the one hand, it argues that indeed the scaling up of the maker movement, for example by becoming part of Fab City ecosystems, is leading to sustainability through resilience and circularity-arguably the two most critical features of future-proof societies. Over the last decade the movement has morphed into a global ecosystem for prototyping software-hardware integration, leading to on-site manufacturing becoming an effective way of reducing carbon footprint. In the process, the maker movement through its humble Fab Lab beginnings is helping to strategically re-generate marginalized areas through progressive re-industrialization [27]. On the other hand is the more balanced view that 3D-printing will not replace mass manufacturing but rather supplement it. Even in the midterm, a mix of technologies and supply chains will be needed. 3D-printing generates significant value in the field of highly personalized goods and to meet demand for smaller quantities at affordable prices. However, it will still be too expensive and cumbersome to meet the demands for very cheap standardized products and, in the context of growing world trade, standardization is critical to keep prices low as well as keep quality and reliability up. Thus, more likely is that manufacturing develops in two phases: the base products will be mass-manufactured, while finalization and personalization will happen in or close to the market, 
both geographically and culturally [28]. Some of these tensions, challenges and indeed opportunities are illustrated in Figure 22. This attempts to capture and summarize much of the evidence and discussion in this paper as to how ICT is being used as the new "general purpose technology" to help "jump the sustainability gap" in how goods are made between the bottom-up, small scale maker movement and the larger city- and global-scales of Industry 4.0.

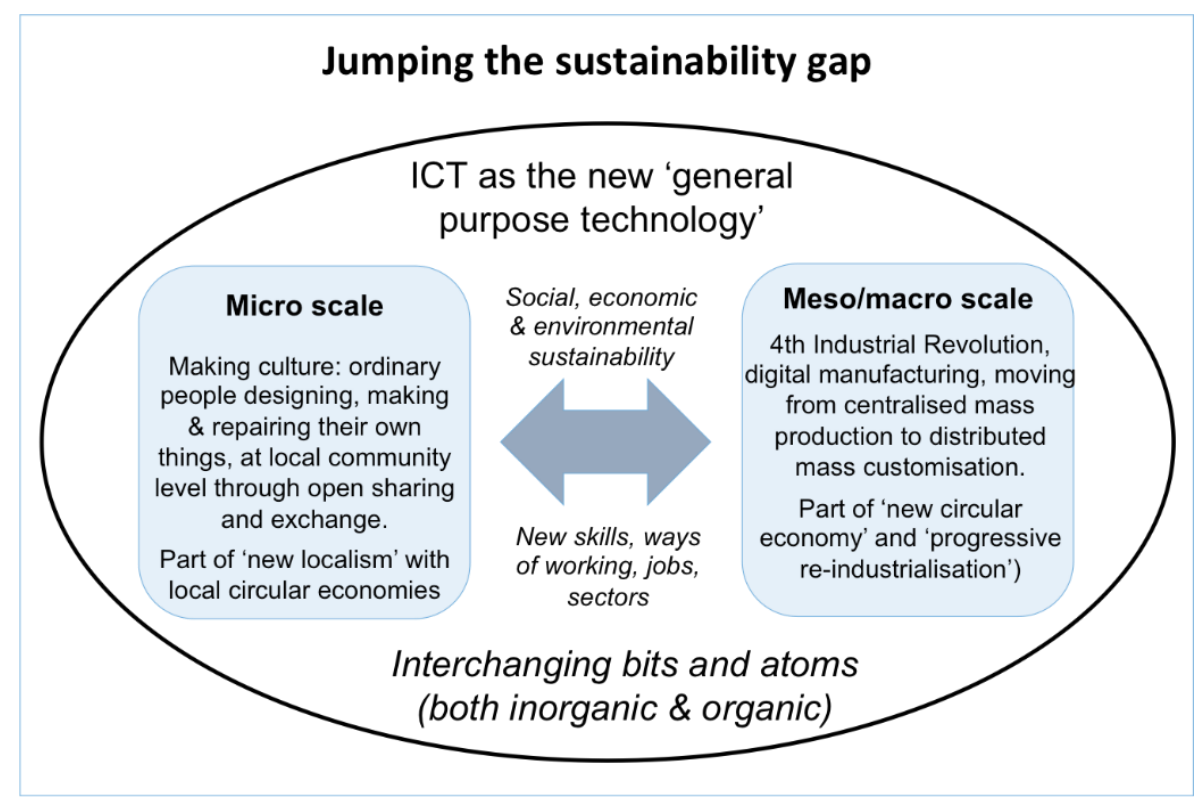

Figure 22. Jumping the sustainability gap.

In conclusion and with feet once again firmly back in the present, it can be confidently stated that the maker movement today is driven more and more at ecosystem and network level with new platforms, service providers and their increasingly strong integration into the value chains of larger companies, many of which are moving towards a distributed manufacturing business model. However, many makers remain as hobbyists, enthusiasts or students and are often amateurs, whilst still being wellsprings of innovation, creating new products and producing value in the community, and some become entrepreneurs and launch start-up companies. Yet, there remains a significant gap between "making", on the one hand, and social and sustainable innovation on the other. Making is often still dominated by the latest gadgets, technical prowess and playful experimentation which are of strategic importance for innovation. However, social and sustainable innovations require stronger connections with communities that have been active-often for decades-improving the living conditions of marginalized people, protecting the environment or caring for older generations. Much of the analysis and many of the cases explored by MAKE-IT are attempting to bridge this gap, but developments for the most part are still at a comparatively early stage.

Author Contributions: Conceptualization, J.M., M.N.S. and E.U.; Data curation, J.M. and S.D.; Formal analysis, J.M., M.N.S. and E.U.; Investigation, J.M., M.N.S. and E.U.; Methodology, J.M., M.N.S., S.D., E.U. and C.V.; Validation, J.M.; Visualization, J.M.; Writing—original draft, J.M., E.U. and C.V.; Writing—review \& editing, J.M., E.U. and C.V.

Funding: This research was funded by European Commission, grant number MAKE-IT -REP-688241-2.

Acknowledgments: Software and technical support: Orfeas K. Katsikis (Danish Technological Institute) and Massimo Menichinelli (The Institute for Advanced Architecture of Catalonia and Fab Lab Barcelona).

Conflicts of Interest: The authors declare no conflicts of interest. 


\section{References}

1. Anderson, C. Makers: The New Industrial Revolution; Random House Business Books: New York, NY, USA, 2012.

2. Rifkin, J. The Zero Marginal Cost Society: The Internet of Things, the Collaborative Commons, and the Eclipse of Capitalism; Palgrave Macmillan: New York, NY, USA, 2014.

3. Gershenfeld, N. How to make almost anything: The digital fabrication revolution. Foreign Aff. 2012, 91, $43-56$.

4. Gershenfeld, N. Designing Reality: How to Survive and Thrive in the Third Digital Revolution; Basic Books: New York, NY, USA, 2017.

5. Charney, D. The Cultural Role(s) of Maker Spaces-Research in Progress; FROM-NOW-ON: London, UK, September 2016; Available online: www.fromnowon.co.uk (accessed on 25 November 2017).

6. Schwab, K. The Fourth Industrial Revolution; World Economic Forum, Penquin Random House: New York, NY, USA, 2016.

7. McAfee, A.; Brynjolfsson, E. Machine Platform Crowd: Harnessing Our Digital Future; W.W. Norton \& Company: New York, NY, USA, 2017.

8. Millard, J. Networks, communities and value chains in digital social innovation for social services. In ICT-Enabled Social Innovation for the European Social Model: A Multi-Disciplinary Reflection and Future Perspectives; Davide, F., Misuraca, G., Eds.; Internet Science, Human-Computer Interaction and Socio-Economics, IOS Press: Amsterdam, The Netherlands, 2018.

9. Sestini, S. Collective awareness platforms: Engines for sustainability and ethics. IEEE Technol. Soc. Mag. 2012, 31, 54-62. [CrossRef]

10. Fourie, I.; Meyer, A. What to make of maker spaces: Tools and DIY only or is there an interconnected information resource space? In Library Hi Tech 33/4; University of Pretoria: Pretoria, South Africa; Emerald Group Publishing Inc.: Bingley, UK, 2015.

11. Millard, J. Towards Sustainable and Resilient Societies: Innovation and Interconnectivity for Social Development; Prepared as a Note by the Secretariat for the United Nations 56th Commission for Social Development; United Nations: New York, NY, USA, 2018; Available online: http:/ / undocs.org/E/CN.5/2018/5 (accessed on 25 November 2017).

12. Nathan, M.; Pratt, A.; Rincon-Aznar, A. Creative Economy Employment in the EU and the UK: A Comparative Analysis; Nesta: London, UK, December 2015; Available online: https: / /www.nesta.org.uk/report/creativeeconomy-employment-in-the-eu-and-uk-a-comparative-analysis (accessed on 25 November 2017).

13. Fujiwara, D.; Dolan, P.; Lawton, R. Creative Occupations and Subjective Wellbeing; Nesta: London, UK, 2015; Available online: http:/ / www.nesta.org.uk/publications/creative-occupations-and-subjective-wellbeing (accessed on 25 November 2017).

14. Bureau of European Policy Advisers (BEPA). Empowering People, Driving Change. Social Innovation in the European Union; European Union: Luxembourg, 2010.

15. Hochgerner, J. Social innovation: The novel creation, variation or new combination of social practices. In Invention, Innovation, and Entrepreneurship; Carayannis, E.G., Ed.; Springer: New York, NY, USA, 2013.

16. Millard, J.; Holtgrewe, U.; Hochgerner, J. Objectives: Social Demands, Societal Challenges and Systemic Change Addressed. In Towards a General Theory and Typology of Social Innovation; Deliverable D1.6 of the SI-DRIVE Project; Technical University of Dortmund: Dortmund, Germany, December 2017; Available online: https: / / www.si-drive.eu (accessed on 25 November 2017).

17. Voigt, C.; Unterfrauner, E.; Stelzer, R. Diversity in FabLabs: Culture, Role Models and the Gendering of Making. In Proceedings of the 4th International Conference, INSCI, Thessaloniki, Greece, 22-24 November 2017; Kompatsiaris, I., Cave, J., Satsiou, A., Georg, C., Passani, A., Kontopoulos, D., McMillan, D., Eds.; Springer: Cham, Switzerland, 2017; pp. 52-68.

18. Kanter, R.M. Men and Women of the Corporation; Basic Books: New York, NY, USA, 1997.

19. Unterfrauner, E.; Shao, J.; Hofer, M.; Fabian, C. The \$environmental value and impact of the Maker movement: Insights from a cross-case analysis of European maker initiatives. J. Clean. Prod. 2018. under review.

20. Buxmann, P.; Hinz, O. Business E Information Systems Engineering; Springer: Basel, Switzerland; Fachmedien Wiesbaden: Wiesbaden, Germany, 2013. 
21. Unterfrauner, E.; Voigt, C. Makers' ambitions to do socially valuable things. Des. J. 2017, 20, 3317-3325. [CrossRef]

22. Menichinelli, M. La dimension économique. In La Révolution est en Marche; Menichinelli, M., Ed.; Editions Pyramyd: Paris, France, 2015.

23. Fab City Global Initiative. Fab City Whitepaper: Locally Productive, Globally Connected Self-Sufficient Cities; Fab City Global Initiative: Barcelona, Spain, 2016; Available online: http:/ / fab.city/whitepaper.pdf (accessed on 25 November 2017).

24. Kirchherr, J.; Reike, D.; Hekkert, M. Conceptualizing the circular economy: An analysis of 114 definitions. Resour. Conserv. Recycl. 2017, 127, 221-232. [CrossRef]

25. Ghisellini, P.; Cialani, C.; Ulgiati, S. A review on circular economy: The expected transition to a balanced interplay of environmental and economic systems. J. Clean. Prod. 2016, 114, 11-32. [CrossRef]

26. Bragon, J.H. Companies that Mimic Life: Leaders of the Emerging Corporate Renaissance; Greenleaf Publishing Limited: Saltaire, UK, 2016.

27. Ermacora, T. Why Maker Spaces Could Be the Secret to Making Smart Cities Smart; World Economic Forum: Cologny, Switzerland, 2018; Available online: https:/ /www.weforum.org/agenda/2018/03/makerspacessmart-sustainable-cities-thomas-ermacora / (accessed on 25 November 2017).

28. Lehmacher, W.; Schwemmer, M. 3D-Printing Might Not Kill Global Trade after All; World Economic Forum: Cologny, Switzerland, 2017; Available online: https:/ / www.weforum.org/agenda/2017/10/3d-printingglobal-trade-supply-chains / (accessed on 25 November 2017).

(C) 2018 by the authors. Licensee MDPI, Basel, Switzerland. This article is an open access article distributed under the terms and conditions of the Creative Commons Attribution (CC BY) license (http://creativecommons.org/licenses/by/4.0/). 